\title{
14. CARBON AND CARBONATE ANALYSES, LEG 16
}

Gerald W. Bode, Scripps Institution of Oceanography, La Jolla, California David S. Cronan, Department of Geology, and University of Ottawa, Ottawa, Canada

The amounts of acid-soluble and acid-insoluble carbon in sediment samples of Leg 16 have been determined in the laboratory of the Deep Sea Drilling Project by Techniques described in detail in Bader, Gerard et al. (1970) and Boyce and Bode (1972). The data are listed in Table 1 and graphically displayed in Chapters 2 through 10 of this volume. In addition, analyses for calcium carbonate were made by a gravimetric method for samples used in connection with the study of the basal iron-rich sediments (Chapter 18 , this volume); the method is described in the last section, and the results have been listed in Table 2 .

TABLE 1

Carbon-Carbonate Analyses, Leg 16 Except as Noted in Key, Analyses Are By LECO 70 Second Analyzer

\begin{tabular}{|c|c|c|c|c|}
\hline $\begin{array}{l}\text { Core, Section } \\
\text { Top of } \\
\text { Interval } \\
\text { (cm) }\end{array}$ & $\begin{array}{l}\text { Depth } \\
\text { in Hole } \\
(\mathrm{m})\end{array}$ & $\begin{array}{c}\text { Carbon } \\
\text { Total } \\
(\%)\end{array}$ & $\begin{array}{c}\text { Organic } \\
\text { Carbon } \\
(\%)\end{array}$ & $\begin{array}{c}\mathrm{CaCO}_{3} \\
(\%)\end{array}$ \\
\hline \multicolumn{5}{|l|}{ DSDP 156} \\
\hline $1-1(139.0)$ & 1.4 & 9.4 & 0.3 & 76 \\
\hline \multicolumn{5}{|l|}{ DSDP 157} \\
\hline $\begin{array}{l}1-1(100.0) \\
1-2(80) \\
1-5(80.0)\end{array}$ & $\begin{array}{l}11.0 \\
12.3 \\
16.8\end{array}$ & $\begin{array}{l}3.3 \\
9.6 \\
9.5\end{array}$ & $\begin{array}{l}0.3 \\
0.4 \\
0.2\end{array}$ & $\begin{array}{l}26 \\
77 \\
78\end{array}$ \\
\hline $\begin{array}{l}2-1(60.0) \\
2-2(60.0) \\
2-3(60.0) \\
2-4(60.0) \\
2-6(60.0)\end{array}$ & $\begin{array}{l}19.6 \\
21.1 \\
22.6 \\
24.1 \\
27.1\end{array}$ & $\begin{array}{l}9.3 \\
9.4 \\
9.6 \\
9.6 \\
9.0\end{array}$ & $\begin{array}{l}0.4 \\
0.4 \\
0.2 \\
0.2 \\
0.3\end{array}$ & $\begin{array}{l}74 \\
75 \\
79 \\
78 \\
72\end{array}$ \\
\hline $\begin{array}{l}3-1(60.0) \\
3-2(60.0) \\
3-3(60.0) \\
3-4(60.0) \\
3-5(60.0) \\
3-6(60.0)\end{array}$ & $\begin{array}{l}28.6 \\
30.1 \\
31.6 \\
33.1 \\
34.6 \\
36.1\end{array}$ & $\begin{array}{l}8.3 \\
8.8 \\
8.4 \\
9.2 \\
8.4 \\
8.0\end{array}$ & $\begin{array}{l}0.5 \\
0.4 \\
0.4 \\
0.3 \\
0.3 \\
0.3\end{array}$ & $\begin{array}{l}65 \\
70 \\
67 \\
74 \\
67 \\
64\end{array}$ \\
\hline $\begin{array}{l}4-1(60.0) \\
4-1(60.0) \\
4-2(60.0) \\
4-3(60.0) \\
4-4(60.0) \\
4-5(60.0) \\
4-6(60.0)\end{array}$ & $\begin{array}{l}37.6 \\
37.6 \\
39.1 \\
40.6 \\
42.1 \\
43.6 \\
45.1\end{array}$ & $\begin{array}{r}10.1 \\
8.9 \\
8.6 \\
7.9 \\
7.9 \\
8.0 \\
7.7\end{array}$ & $\begin{array}{l}0.2 \\
0.3 \\
0.3 \\
0.3 \\
0.3 \\
0.3 \\
0.3\end{array}$ & $\begin{array}{l}83 \\
72 \\
69 \\
63 \\
63 \\
64 \\
62\end{array}$ \\
\hline $\begin{array}{l}5-1(60.0) \\
5-2(60.0) \\
5-3(60.0) \\
5-4(60.0) \\
5-5(60.0) \\
5-6(60.0)\end{array}$ & $\begin{array}{l}46.6 \\
48.1 \\
49.6 \\
51.1 \\
52.6 \\
54.1\end{array}$ & $\begin{array}{l}9.2 \\
9.0 \\
9.5 \\
9.5 \\
9.2 \\
9.0\end{array}$ & $\begin{array}{l}0.0 \\
0.4 \\
0.2 \\
0.2 \\
0.3 \\
0.3\end{array}$ & $\begin{array}{l}77 \\
72 \\
77 \\
77 \\
74 \\
73\end{array}$ \\
\hline $\begin{array}{l}6-1(60.0) \\
6-2(60.0)\end{array}$ & $\begin{array}{l}55.6 \\
57.1\end{array}$ & $\begin{array}{l}7.3 \\
7.2\end{array}$ & $\begin{array}{l}0.4 \\
0.4\end{array}$ & $\begin{array}{l}58 \\
56\end{array}$ \\
\hline
\end{tabular}

TABLE 1 - Continued

\begin{tabular}{|c|c|c|c|c|}
\hline $\begin{array}{l}\text { Core, Section } \\
\text { Top of } \\
\text { Interval } \\
\text { (cm) }\end{array}$ & $\begin{array}{l}\text { Depth } \\
\text { in Hole } \\
(\mathrm{m})\end{array}$ & $\begin{array}{c}\text { Carbon } \\
\text { Total } \\
(\%)\end{array}$ & $\begin{array}{c}\text { Organic } \\
\text { Carbon } \\
(\%)\end{array}$ & $\begin{array}{c}\mathrm{CaCO}_{3} \\
(\%)\end{array}$ \\
\hline $\begin{array}{l}6-3(60.0) \\
6-4(60.0) \\
6-5(60.0) \\
6-6(60.0)\end{array}$ & $\begin{array}{l}58.6 \\
60.1 \\
61.6 \\
63.1\end{array}$ & $\begin{array}{l}7.7 \\
8.8 \\
7.5 \\
8.1\end{array}$ & $\begin{array}{l}0.3 \\
0.6 \\
0.5 \\
0.4\end{array}$ & $\begin{array}{l}61 \\
68 \\
59 \\
64\end{array}$ \\
\hline $\begin{array}{l}7-1(100.0) \\
7-2(60.0) \\
7-3(60.0) \\
7-4(60.0) \\
7-5(60.0)\end{array}$ & $\begin{array}{l}65.0 \\
66.1 \\
67.6 \\
69.1 \\
70.6\end{array}$ & $\begin{array}{l}8.5 \\
8.6 \\
9.0 \\
8.8 \\
8.3\end{array}$ & $\begin{array}{l}0.4 \\
0.3 \\
0.2 \\
0.2 \\
0.3\end{array}$ & $\begin{array}{l}68 \\
69 \\
73 \\
71 \\
67\end{array}$ \\
\hline $\begin{array}{l}8-1(60.0) \\
8-2(60.0) \\
8-3(60.0) \\
8-4(60.0)\end{array}$ & $\begin{array}{l}73.6 \\
75.1 \\
76.6 \\
78.1\end{array}$ & $\begin{array}{l}8.9 \\
8.6 \\
8.2 \\
8.2\end{array}$ & $\begin{array}{l}0.2 \\
0.3 \\
0.3 \\
0.3\end{array}$ & $\begin{array}{l}72 \\
69 \\
66 \\
66\end{array}$ \\
\hline $\begin{array}{l}9-1(120.0) \\
9-2(60.0) \\
9-3(60.0) \\
9-4(60.0) \\
9-5(60.0) \\
9-6(60.0)\end{array}$ & $\begin{array}{l}83.2 \\
84.1 \\
85.6 \\
87.1 \\
88.6 \\
90.1\end{array}$ & $\begin{array}{l}8.5 \\
7.4 \\
8.1 \\
6.8 \\
7.1 \\
6.7\end{array}$ & $\begin{array}{l}1.1 \\
2.0 \\
1.2 \\
1.4 \\
1.5 \\
1.3\end{array}$ & $\begin{array}{l}62 \\
44 \\
57 \\
45 \\
47 \\
45\end{array}$ \\
\hline $\begin{array}{l}10-1(60.0) \\
10-2(60.0) \\
10-3(60.0) \\
10-4(60.0)\end{array}$ & $\begin{array}{l}91.6 \\
93.1 \\
94.6 \\
96.1\end{array}$ & $\begin{array}{l}6.9 \\
7.4 \\
7.1 \\
7.4\end{array}$ & $\begin{array}{l}1.1 \\
1.6 \\
1.6 \\
1.3\end{array}$ & $\begin{array}{l}48 \\
48 \\
46 \\
50\end{array}$ \\
\hline $\begin{array}{l}11-1(70.0) \\
11-2(60.0) \\
11-3(30.0) \\
11-4(60.0)\end{array}$ & $\begin{array}{r}99.7 \\
101.1 \\
102.3 \\
104.1\end{array}$ & $\begin{array}{l}8.3 \\
8.6 \\
9.0 \\
8.8\end{array}$ & $\begin{array}{l}0.4 \\
0.6 \\
0.6 \\
0.6\end{array}$ & $\begin{array}{l}65 \\
67 \\
70 \\
68\end{array}$ \\
\hline $\begin{array}{l}13-1(60.0) \\
13-2(60.0) \\
13-3(10.0) \\
13-4(60.0) \\
13-6(60.0)\end{array}$ & $\begin{array}{l}117.6 \\
119.1 \\
120.1 \\
122.1 \\
125.1\end{array}$ & $\begin{array}{l}9.0 \\
9.2 \\
9.3 \\
7.6 \\
8.4\end{array}$ & $\begin{array}{l}0.3 \\
0.3 \\
0.3 \\
0.8 \\
0.7\end{array}$ & $\begin{array}{l}72 \\
74 \\
75 \\
56 \\
64\end{array}$ \\
\hline $\begin{array}{l}14-1(100.0) \\
14-2(60.0) \\
14-3(60.0) \\
14-4(60.0) \\
14-5(60.0) \\
14-6(60.0)\end{array}$ & $\begin{array}{l}127.0 \\
128.1 \\
129.6 \\
131.1 \\
132.6 \\
134.1\end{array}$ & $\begin{array}{l}8.4 \\
7.0 \\
5.9 \\
7.6 \\
6.7 \\
8.0\end{array}$ & $\begin{array}{l}0.4 \\
0.9 \\
0.9 \\
0.5 \\
0.3 \\
0.5\end{array}$ & $\begin{array}{l}66 \\
50 \\
42 \\
59 \\
53 \\
63\end{array}$ \\
\hline $\begin{array}{l}15-1(60.0) \\
15-2(60.0) \\
15-3(60.0) \\
15-3(60.0) \\
15-4(60.0) \\
15-5(60.0) \\
15-6(60.0)\end{array}$ & $\begin{array}{l}135.6 \\
137.1 \\
138.6 \\
138.6 \\
140.1 \\
141.6 \\
143.1\end{array}$ & $\begin{array}{l}7.2 \\
7.6 \\
8.4 \\
7.8 \\
8.4 \\
8.4 \\
7.9\end{array}$ & $\begin{array}{l}0.6 \\
0.5 \\
0.3 \\
0.3 \\
0.3 \\
0.3 \\
0.3\end{array}$ & $\begin{array}{l}55 \\
59 \\
68 \\
62 \\
67 \\
67 \\
63\end{array}$ \\
\hline $\begin{array}{l}16-1(60.0) \\
16-2(60.0) \\
16-3(60.0) \\
16-4(60.0) \\
16-5(60.0) \\
16-6(60.0)\end{array}$ & $\begin{array}{l}144.6 \\
146.1 \\
147.6 \\
149.1 \\
150.6 \\
152.1\end{array}$ & $\begin{array}{l}7.5 \\
8.0 \\
7.8 \\
8.0 \\
7.7 \\
6.8\end{array}$ & $\begin{array}{l}0.3 \\
0.3 \\
0.3 \\
0.3 \\
0.2 \\
0.2\end{array}$ & $\begin{array}{l}60 \\
65 \\
62 \\
64 \\
62 \\
55\end{array}$ \\
\hline
\end{tabular}


TABLE 1 - Continued

\begin{tabular}{|c|c|c|c|c|}
\hline $\begin{array}{l}\text { Core, Section } \\
\text { Top of } \\
\text { Interval } \\
\text { (cm) }\end{array}$ & $\begin{array}{l}\text { Depth } \\
\text { in Hole } \\
(\mathrm{m})\end{array}$ & $\begin{array}{l}\text { Carbon } \\
\text { Total } \\
(\%)\end{array}$ & $\begin{array}{c}\text { Organic } \\
\text { Carbon } \\
(\%)\end{array}$ & $\begin{array}{c}\mathrm{CaCO}_{3} \\
(\%)\end{array}$ \\
\hline $17-1(60.0)$ & 153.6 & 7.4 & 0.8 & 55 \\
\hline $17-2(60.0)$ & 155.1 & 7.7 & 0.4 & 62 \\
\hline $17-3(60.0)$ & 156.6 & 7.9 & 0.3 & 63 \\
\hline $17-4(60.0)$ & 158.1 & 7.6 & 0.4 & 60 \\
\hline $17-5(60.0)$ & 159.6 & 7.3 & 0.5 & 57 \\
\hline $17-6(60.0)$ & 161.1 & 6.9 & 0.6 & 53 \\
\hline $18-1(63.0)$ & 162.6 & 7.0 & 0.6 & 53 \\
\hline $18-2(60.0)$ & 164.1 & 6.9 & 0.5 & 54 \\
\hline $18-3(60.0)$ & 165.6 & 7.4 & 0.4 & 59 \\
\hline $18-4(61.0)$ & 167.1 & 7.6 & 0.3 & 61 \\
\hline $18-5(60.0)$ & 168.6 & 7.6 & 0.4 & 60 \\
\hline $18-6(60.0)$ & 170.1 & 7.5 & 0.4 & 59 \\
\hline $19-1(60.0)$ & 171.6 & 8.1 & 0.7 & 62 \\
\hline $19-2(60.0)$ & 173.1 & 8.8 & 0.6 & 69 \\
\hline $19-3(60.0)$ & 174.6 & 8.2 & 0.5 & 64 \\
\hline $19-4(60.0)$ & 176.1 & 9.3 & 0.9 & 70 \\
\hline $19-5(60.0)$ & 177.6 & 7.2 & 0.6 & 56 \\
\hline $19-6(60.0)$ & 179.1 & 6.4 & 0.8 & 47 \\
\hline $20-1(120.0)$ & $\begin{array}{l}181.2 \\
182.1\end{array}$ & $\begin{array}{l}8.2 \\
8.0\end{array}$ & $\begin{array}{l}0.5 \\
0.5\end{array}$ & 64 \\
\hline $20-2(60.0)$ & 182.1 & 8.0 & 0.5 & 63 \\
\hline $21-1(60.0)$ & 189.6 & 7.9 & 0.4 & 62 \\
\hline $21-2(60.0)$ & 191.1 & 7.3 & 0.5 & 57 \\
\hline $21-3(60.0)$ & 192.6 & 7.6 & 0.5 & 60 \\
\hline $21-4(60.0)$ & 194.1 & 7.2 & 0.5 & 56 \\
\hline $21-5(60.0)$ & 195.6 & 7.1 & 0.6 & 54 \\
\hline $21-6(60.0)$ & 197.1 & 7.0 & 0.5 & 54 \\
\hline $22-1(60.0)$ & 198.6 & 7.4 & 0.5 & 58 \\
\hline $22-2(30.0)$ & 199.8 & 7.0 & 0.4 & 54 \\
\hline $22-3(60.0)$ & 201.6 & 7.1 & 0.4 & 55 \\
\hline $22-4(60.0)$ & 203.1 & 7.6 & 0.4 & 61 \\
\hline $22-5(60.0)$ & 204.6 & 7.9 & 0.4 & 63 \\
\hline $22-6(60.0)$ & 206.1 & 7.9 & 0.5 & 62 \\
\hline $23-1(60.0)$ & 207.6 & 8.5 & 0.3 & 68 \\
\hline $23-2(60.0)$ & 209.1 & 7.8 & 0.4 & 62 \\
\hline $23-3(60.0)$ & 210.6 & 7.4 & 0.4 & 59 \\
\hline $23-4(60.0)$ & 212.1 & 8.7 & 0.4 & 69 \\
\hline $23-5(60.0)$ & 213.6 & 8.8 & 0.3 & 71 \\
\hline $23-6(60.0)$ & 215.1 & 9.3 & 0.2 & 75 \\
\hline $24-1(60.0)$ & 216.6 & 9.1 & 0.4 & 73 \\
\hline $24-2(60.0)$ & 218.1 & 8.6 & 0.4 & 68 \\
\hline $24-3(60.0)$ & 219.6 & 9.2 & 0.3 & 75 \\
\hline $24-4(60.0)$ & 221.1 & 9.4 & 0.3 & 76 \\
\hline $25-2(60.0)$ & 227.1 & 8.7 & 0.5 & 69 \\
\hline $25-3(60.0)$ & 228.6 & 8.8 & 0.3 & 70 \\
\hline $25-4(60.0)$ & 230.1 & 8.7 & 0.3 & 70 \\
\hline $25-5(60.0)$ & 231.6 & 8.4 & 0.3 & 68 \\
\hline $26-1(60.0)$ & 234.6 & 8.6 & 0.3 & 69 \\
\hline $26-2(60.0)$ & 236.1 & 9.8 & 0.2 & 80 \\
\hline $26-3(60.0)$ & 237.6 & 9.7 & 0.2 & 78 \\
\hline $26-4(60.0)$ & 239.1 & 9.3 & 0.2 & 76 \\
\hline $26-5(60.0)$ & 240.6 & 8.6 & 0.4 & 68 \\
\hline $26-6(60.0)$ & 242.1 & 7.8 & 0.2 & 64 \\
\hline $27-1(60.0)$ & 243.6 & 8.3 & 0.3 & 67 \\
\hline $27-2(60.0)$ & 245.1 & 8.3 & 0.2 & 67 \\
\hline $27-3(60.0)$ & 246.6 & 8.7 & 0.3 & 70 \\
\hline $27-4(60.0)$ & 248.1 & 8.7 & 0.2 & 70 \\
\hline $27-5(60.0)$ & 249.6 & 9.1 & 0.2 & 74 \\
\hline $27-6(60.0)$ & 251.1 & 7.5 & 0.2 & 61 \\
\hline $28-1(60.0)$ & 252.6 & 8.0 & 0.3 & 65 \\
\hline $28-2(60.0)$ & 254.1 & 9.1 & 0.2 & 74 \\
\hline $28-3(60.0)$ & 255.6 & 9.6 & 0.2 & 78 \\
\hline $28-4(60.0)$ & 257.1 & 9.9 & 0.2 & 81 \\
\hline
\end{tabular}

TABLE 1 - Continued

\begin{tabular}{|c|c|c|c|c|}
\hline $\begin{array}{l}\text { Core, Section } \\
\text { Top of } \\
\text { Interval } \\
\text { (cm) }\end{array}$ & $\begin{array}{l}\text { Depth } \\
\text { in Hole } \\
\text { (m) }\end{array}$ & $\begin{array}{c}\text { Carbon } \\
\text { Total } \\
(\%)\end{array}$ & $\begin{array}{c}\text { Organic } \\
\text { Carbon } \\
(\%)\end{array}$ & $\begin{array}{c}\mathrm{CaCO}_{3} \\
(\%)\end{array}$ \\
\hline $\begin{array}{l}28-5(60.0) \\
28-6(60.0)\end{array}$ & $\begin{array}{l}258.6 \\
260.1\end{array}$ & $\begin{array}{l}9.7 \\
9.6\end{array}$ & $\begin{array}{l}0.2 \\
0.2\end{array}$ & $\begin{array}{l}79 \\
79\end{array}$ \\
\hline $\begin{array}{l}29-1(100.0) \\
29-2(60.0) \\
29-3(60.0)\end{array}$ & $\begin{array}{l}262.0 \\
263.1 \\
264.6\end{array}$ & $\begin{array}{r}9.3 \\
8.9 \\
10.0\end{array}$ & $\begin{array}{l}0.3 \\
0.3 \\
0.2\end{array}$ & $\begin{array}{l}75 \\
72 \\
81\end{array}$ \\
\hline $\begin{array}{l}30-1(60.0) \\
30-2(60.0) \\
30-3(60.0) \\
30-4(50.0) \\
30-5(60.0) \\
30-6(60.0)\end{array}$ & $\begin{array}{l}270.6 \\
272.1 \\
273.6 \\
275.0 \\
276.6 \\
278.1\end{array}$ & $\begin{array}{l}9.3 \\
8.7 \\
9.0 \\
8.6 \\
8.4 \\
9.0\end{array}$ & $\begin{array}{l}0.3 \\
0.3 \\
0.2 \\
0.3 \\
0.3 \\
0.1\end{array}$ & $\begin{array}{l}74 \\
70 \\
73 \\
69 \\
67 \\
73\end{array}$ \\
\hline $\begin{array}{l}31-1(105.0) \\
31-2(60.0) \\
31-3(60.0) \\
31-4(60.0) \\
31-5(60.0) \\
31-6(50.0)\end{array}$ & $\begin{array}{l}280.0 \\
281.1 \\
282.6 \\
284.1 \\
285.6 \\
287.0\end{array}$ & $\begin{array}{l}9.2 \\
9.4 \\
8.6 \\
9.4 \\
9.2 \\
9.0\end{array}$ & $\begin{array}{l}0.2 \\
0.2 \\
0.3 \\
0.2 \\
0.2 \\
0.3\end{array}$ & $\begin{array}{l}75 \\
77 \\
69 \\
77 \\
75 \\
73\end{array}$ \\
\hline $\begin{array}{l}32-1(60.0) \\
32-2(60.0) \\
32-3(60.0) \\
32-4(60.0) \\
32-5(60.0) \\
32-6(60.0)\end{array}$ & $\begin{array}{l}288.6 \\
290.1 \\
291.6 \\
293.1 \\
294.6 \\
296.1\end{array}$ & $\begin{array}{r}9.4 \\
8.8 \\
9.0 \\
9.8 \\
9.8 \\
10.0\end{array}$ & $\begin{array}{l}0.2 \\
0.3 \\
0.3 \\
0.2 \\
0.2 \\
0.2\end{array}$ & $\begin{array}{l}77 \\
71 \\
72 \\
80 \\
80 \\
82\end{array}$ \\
\hline $\begin{array}{l}33-1(60.0) \\
33-2(60.0) \\
33-3(60.0) \\
33-4(60.0) \\
33-5(60.0) \\
33-6(60.0)\end{array}$ & $\begin{array}{l}297.6 \\
299.1 \\
300.6 \\
302.1 \\
303.6 \\
305.1\end{array}$ & $\begin{array}{l}9.2 \\
7.6 \\
6.8 \\
9.0 \\
8.7 \\
8.8\end{array}$ & $\begin{array}{l}0.3 \\
0.3 \\
0.2 \\
0.3 \\
0.2 \\
0.2\end{array}$ & $\begin{array}{l}74 \\
60 \\
54 \\
72 \\
71 \\
71\end{array}$ \\
\hline $35-1(60.0)$ & 315.6 & 9.0 & 0.3 & 73 \\
\hline $\begin{array}{l}36-1(100.0) \\
36-2(60.0) \\
36-3(60.0)\end{array}$ & $\begin{array}{l}325.0 \\
326.1 \\
327.6\end{array}$ & $\begin{array}{r}9.9 \\
9.7 \\
10.0\end{array}$ & $\begin{array}{l}0.2 \\
0.2 \\
0.2\end{array}$ & $\begin{array}{l}81 \\
78 \\
81\end{array}$ \\
\hline $42-1(70.0)$ & 368.7 & 10.9 & 0.2 & 89 \\
\hline \multicolumn{5}{|l|}{ DSDP 157A } \\
\hline $\begin{array}{l}1-1(100.0) \\
1-2(30.0) \\
1-3(60.0) \\
1-4(60.0) \\
1-5(60.0) \\
1-6(60.0)\end{array}$ & $\begin{array}{l}1.0 \\
1.8 \\
3.6 \\
5.1 \\
6.6 \\
8.1\end{array}$ & $\begin{array}{l}9.9 \\
9.2 \\
8.8 \\
8.3 \\
9.0 \\
8.5\end{array}$ & $\begin{array}{l}0.8 \\
0.6 \\
0.7 \\
0.4 \\
0.5 \\
0.3\end{array}$ & $\begin{array}{l}76 \\
72 \\
67 \\
66 \\
71 \\
68\end{array}$ \\
\hline $2-1(60.0)$ & 9.6 & 8.5 & 0.3 & 68 \\
\hline $\begin{array}{l}3-1(30.0) \\
3-2(114.0) \\
3-3(60.0) \\
3-4(60.0) \\
3-5(70.0) \\
3-6(60.0)\end{array}$ & $\begin{array}{l}18.3 \\
20.6 \\
21.6 \\
23.1 \\
24.7 \\
26.1\end{array}$ & $\begin{array}{l}9.0 \\
8.8 \\
8.5 \\
8.8 \\
8.5 \\
9.0\end{array}$ & $\begin{array}{l}0.2 \\
0.3 \\
0.2 \\
0.3 \\
0.3 \\
0.3\end{array}$ & $\begin{array}{l}73 \\
71 \\
69 \\
70 \\
68 \\
73\end{array}$ \\
\hline
\end{tabular}

DSDP 158

\begin{tabular}{lrlll}
$1-1(60.0)$ & 0.6 & 6.7 & 0.7 & 51 \\
$1-2(60.0)$ & 2.1 & 6.0 & 0.6 & 46 \\
$1-3(60.0)$ & 3.6 & 6.9 & 0.6 & 52 \\
$2-1(60.0)$ & 9.6 & 6.0 & 0.8 & 43 \\
$2-2(60.0)$ & 11.1 & 6.7 & 0.6 & 51 \\
$2-3(60.0)$ & 12.6 & 6.6 & 0.5 & 51 \\
$2-4(60.0)$ & 14.1 & 7.2 & 0.5 & 56 \\
$2-5(60.0)$ & 15.6 & 5.9 & 0.5 & 45 \\
\hline
\end{tabular}


TABLE 1 - Continued

\begin{tabular}{|c|c|c|c|c|}
\hline $\begin{array}{l}\text { Core, Section } \\
\text { Top of } \\
\text { Interval } \\
\text { (cm) }\end{array}$ & $\begin{array}{l}\text { Depth } \\
\text { in Hole } \\
\text { (m) }\end{array}$ & $\begin{array}{c}\text { Carbon } \\
\text { Total } \\
(\%)\end{array}$ & $\begin{array}{c}\text { Organic } \\
\text { Carbon } \\
(\%)\end{array}$ & $\begin{array}{c}\mathrm{CaCO}_{3} \\
(\%)\end{array}$ \\
\hline $3-1(60.0)$ & 18.6 & 7.0 & 0.3 & 56 \\
\hline $3-2(60.0)$ & 20.1 & 3.7 & 0.2 & 30 \\
\hline $3-3(60.0)$ & 21.6 & 7.9 & 0.4 & 62 \\
\hline $3-4(60.0)$ & 23.1 & 6.8 & 0.3 & 54 \\
\hline $3-5(60.0)$ & 24.6 & 8.2 & 0.3 & 66 \\
\hline $3-6(60.0)$ & 26.1 & 7.8 & 0.3 & 63 \\
\hline $4-1(60.0)$ & 27.6 & 8.1 & 0.3 & 65 \\
\hline $4-2(60.0)$ & 29.1 & 9.0 & 0.2 & 73 \\
\hline $4-3(60.0)$ & 30.6 & 8.8 & 0.2 & 72 \\
\hline $4-4(60.0)$ & 32.1 & 9.2 & 0.3 & 75 \\
\hline $5-1(60.0)$ & 36.6 & 10.1 & 0.2 & 83 \\
\hline $5-2(60.0)$ & 38.1 & 9.6 & 0.2 & 78 \\
\hline $5-3(60.0)$ & 39.6 & 9.9 & 0.2 & 81 \\
\hline $5-4(60.0)$ & 41.1 & 9.7 & 0.2 & 80 \\
\hline $5-5(60.0)$ & 42.6 & 9.9 & 0.2 & 81 \\
\hline $5-6(60.0)$ & 44.1 & 9.7 & 0.2 & 79 \\
\hline $6-1(60.0)$ & 45.6 & 9.9 & 0.2 & 81 \\
\hline $6-2(60.0)$ & 47.1 & 9.9 & 0.2 & 81 \\
\hline $6-3(60.0)$ & 48.6 & 11.7 & 0.2 & 96 \\
\hline $6-4(60.0)$ & 50.1 & 10.3 & 0.2 & 84 \\
\hline $6-5(60.0)$ & 51.6 & 8.2 & 0.3 & 66 \\
\hline $6-6(60.0)$ & 53.1 & 8.5 & 0.2 & 69 \\
\hline $7-1(60.0)$ & 54.6 & 9.8 & 0.2 & 81 \\
\hline $7-2(60.0)$ & 56.1 & 10.6 & 0.2 & 87 \\
\hline $7-3(60.0)$ & 57.6 & 10.4 & 0.2 & 85 \\
\hline $7-4(60.0)$ & 59.1 & 10.8 & 0.2 & 88 \\
\hline $7-5(60.0)$ & 60.6 & 8.0 & 0.2 & 65 \\
\hline $7-6(60.0)$ & 62.1 & 10.4 & 0.2 & 85 \\
\hline $8-1(60.0)$ & 63.6 & 10.8 & 0.2 & 88 \\
\hline $8-2(60.0)$ & 65.1 & 10.5 & 0.2 & 86 \\
\hline $8-3(60.0)$ & 66.6 & 10.6 & 0.2 & 87 \\
\hline $8-4(60.0)$ & 68.1 & 10.8 & 0.2 & 88 \\
\hline $8-5(60.0)$ & 69.6 & 9.3 & 0.2 & 76 \\
\hline $8-6(60.0)$ & 71.1 & 10.6 & 0.2 & 86 \\
\hline $9-1(60.0)$ & 72.6 & 10.3 & 0.2 & 84 \\
\hline $9-2(60.0)$ & 74.1 & 10.5 & 0.2 & 86 \\
\hline $9-3(60.0)$ & 75.6 & 10.2 & 0.2 & 84 \\
\hline $9-4(60.0)$ & 77.1 & 10.1 & 0.2 & 83 \\
\hline $9-5(60.0)$ & 78.6 & 10.1 & 0.2 & 82 \\
\hline $9-6(60.0)$ & 80.1 & 9.9 & 0.3 & 81 \\
\hline $10-1(60.0)$ & 81.6 & 10.7 & 0.3 & 87 \\
\hline $10-2(60.0)$ & 83.1 & 10.2 & 0.3 & 83 \\
\hline $10-3(60.0)$ & 84.6 & 10.8 & 0.3 & 88 \\
\hline $10-4(60.0)$ & 86.1 & 10.5 & 0.2 & 86 \\
\hline $10-5(60.0)$ & 87.6 & 10.4 & 0.2 & 85 \\
\hline $10-6(60.0)$ & 89.1 & 10.6 & 0.2 & 86 \\
\hline $11-1(60.0)$ & 90.6 & 10.4 & 0.3 & 84 \\
\hline $11-2(60.0)$ & 92.1 & 10.5 & 0.3 & 85 \\
\hline $11-3(60.0)$ & 93.6 & 10.4 & 0.3 & 84 \\
\hline $11-4(60.0)$ & 95.1 & 10.8 & 0.3 & 87 \\
\hline $11-5(60.0)$ & 96.6 & 9.5 & 0.2 & 77 \\
\hline $11-6(60.0)$ & 98.1 & 9.4 & 0.3 & 76 \\
\hline $12-1(60.0)$ & 99.6 & 10.2 & 0.3 & 82 \\
\hline $12-2(60.0)$ & 101.1 & 10.5 & 0.2 & 86 \\
\hline $12-3(60.0)$ & 102.6 & 10.3 & 0.2 & 85 \\
\hline $12-4(60.0)$ & 104.1 & 10.5 & 0.2 & 86 \\
\hline $12-5(60.0)$ & 105.6 & 10.7 & 0.3 & 86 \\
\hline $12-6(60.0)$ & 107.1 & 10.5 & 0.2 & 86 \\
\hline $13-1(60.0)$ & 108.6 & 10.4 & 0.4 & 83 \\
\hline $13-2(60.0)$ & 110.1 & 10.8 & 0.3 & 88 \\
\hline $13-4(60.0)$ & 113.1 & 10.7 & 0.2 & 88 \\
\hline
\end{tabular}

TABLE 1 - Continued

\begin{tabular}{|c|c|c|c|c|}
\hline $\begin{array}{l}\text { Core, Section } \\
\text { Top of } \\
\text { Interval } \\
\text { (cm) }\end{array}$ & $\begin{array}{l}\text { Depth } \\
\text { in Hole } \\
(\mathrm{m})\end{array}$ & $\begin{array}{c}\text { Carbon } \\
\text { Total } \\
(\%)\end{array}$ & $\begin{array}{c}\text { Organic } \\
\text { Carbon } \\
(\%)\end{array}$ & $\begin{array}{c}\mathrm{CaCO}_{3} \\
(\%)\end{array}$ \\
\hline $\begin{array}{l}13-5(60.0) \\
13-6(60.0)\end{array}$ & $\begin{array}{l}114.6 \\
116.1\end{array}$ & $\begin{array}{l}10.8 \\
10.6\end{array}$ & $\begin{array}{l}0.3 \\
0.2\end{array}$ & $\begin{array}{l}87 \\
87\end{array}$ \\
\hline $\begin{array}{l}14-1(50.0) \\
14-2(60.0) \\
14-3(60.0) \\
14-4(60.0) \\
14-5(60.0) \\
14-6(60.0)\end{array}$ & $\begin{array}{l}117.5 \\
119.1 \\
120.6 \\
122.1 \\
123.6 \\
125.1\end{array}$ & $\begin{array}{r}10.2 \\
10.1 \\
10.2 \\
10.1 \\
9.5 \\
9.4\end{array}$ & $\begin{array}{l}0.3 \\
0.4 \\
0.4 \\
0.4 \\
0.4 \\
0.5\end{array}$ & $\begin{array}{l}82 \\
81 \\
82 \\
81 \\
76 \\
74\end{array}$ \\
\hline $\begin{array}{l}15-1(60.0) \\
15-2(60.0) \\
15-4(60.0) \\
15-5(60.0) \\
15-6(60.0)\end{array}$ & $\begin{array}{l}126.6 \\
128.1 \\
131.1 \\
132.6 \\
134.1\end{array}$ & $\begin{array}{l}9.8 \\
9.6 \\
8.6 \\
9.7 \\
8.5\end{array}$ & $\begin{array}{l}0.2 \\
0.2 \\
0.3 \\
0.9 \\
0.3\end{array}$ & $\begin{array}{l}80 \\
78 \\
69 \\
73 \\
69\end{array}$ \\
\hline $\begin{array}{l}16-1(60.0) \\
16-2(60.0) \\
16-3(60.0) \\
16-4(60.0) \\
16-5(60.0) \\
16-6(60.0)\end{array}$ & $\begin{array}{l}135.6 \\
137.1 \\
138.6 \\
140.1 \\
141.6 \\
143.1\end{array}$ & $\begin{array}{r}9.8 \\
10.2 \\
9.7 \\
10.2 \\
10.6 \\
10.0\end{array}$ & $\begin{array}{l}0.4 \\
0.2 \\
0.2 \\
0.2 \\
0.2 \\
0.2\end{array}$ & $\begin{array}{l}78 \\
84 \\
79 \\
83 \\
87 \\
82\end{array}$ \\
\hline $\begin{array}{l}17-1(60.0) \\
17-2(60.0) \\
17-3(60.0) \\
17-4(60.0) \\
17-5(60.0) \\
17-6(60.0)\end{array}$ & $\begin{array}{l}144.6 \\
146.1 \\
147.6 \\
149.1 \\
150.6 \\
152.1\end{array}$ & $\begin{array}{l}9.8 \\
9.7 \\
9.9 \\
9.8 \\
9.5 \\
8.7\end{array}$ & $\begin{array}{l}0.2 \\
0.2 \\
0.2 \\
0.2 \\
0.2 \\
0.3\end{array}$ & $\begin{array}{l}79 \\
79 \\
80 \\
80 \\
77 \\
70\end{array}$ \\
\hline $\begin{array}{l}18-1(60.0) \\
18-2(60.0) \\
18-3(60.0) \\
18-4(60.0) \\
18-5(60.0) \\
18-6(60.0)\end{array}$ & $\begin{array}{l}153.6 \\
155.1 \\
156.6 \\
158.1 \\
159.6 \\
161.1\end{array}$ & $\begin{array}{l}9.0 \\
8.9 \\
6.9 \\
8.5 \\
7.7 \\
8.4\end{array}$ & $\begin{array}{l}0.3 \\
0.2 \\
0.2 \\
0.3 \\
0.2 \\
0.2\end{array}$ & $\begin{array}{l}72 \\
72 \\
56 \\
69 \\
63 \\
68\end{array}$ \\
\hline $\begin{array}{l}19-1(60.0) \\
19-2(60.0) \\
19-3(60.0) \\
19-4(60.0) \\
19-5(100.0) \\
19-6(60.0)\end{array}$ & $\begin{array}{l}162.6 \\
164.1 \\
165.6 \\
167.1 \\
169.0 \\
170.1\end{array}$ & $\begin{array}{r}9.3 \\
9.6 \\
8.5 \\
9.5 \\
10.5 \\
10.6\end{array}$ & $\begin{array}{l}0.3 \\
0.2 \\
0.3 \\
0.2 \\
0.2 \\
0.1\end{array}$ & $\begin{array}{l}75 \\
78 \\
68 \\
77 \\
86 \\
88\end{array}$ \\
\hline $\begin{array}{l}20-1(60.0) \\
20-2(60.0) \\
20-3(60.0) \\
20-4(60.0) \\
20-5(50.0) \\
20-6(60.0)\end{array}$ & $\begin{array}{l}171.6 \\
173.1 \\
174.6 \\
176.1 \\
177.5 \\
179.1\end{array}$ & $\begin{array}{l}10.6 \\
10.6 \\
10.5 \\
10.3 \\
10.1 \\
10.5\end{array}$ & $\begin{array}{l}0.1 \\
0.2 \\
0.2 \\
0.2 \\
0.2 \\
0.2\end{array}$ & $\begin{array}{l}88 \\
87 \\
86 \\
84 \\
83 \\
86\end{array}$ \\
\hline $\begin{array}{l}21-2(60.0) \\
21-2(60.0) \\
21-3(60.0) \\
21-4(60.0) \\
21-5(60.0)\end{array}$ & $\begin{array}{l}180.6 \\
182.1 \\
183.6 \\
185.1 \\
186.6\end{array}$ & $\begin{array}{r}10.4 \\
10.6 \\
9.9 \\
10.3 \\
10.4\end{array}$ & $\begin{array}{l}0.2 \\
0.2 \\
0.2 \\
0.2 \\
0.1\end{array}$ & $\begin{array}{l}85 \\
87 \\
81 \\
85 \\
85\end{array}$ \\
\hline $\begin{array}{l}22-2(60.0) \\
22-3(60.0) \\
22-4(60.0) \\
22-5(60.0) \\
22-6(60.0)\end{array}$ & $\begin{array}{l}191.1 \\
192.6 \\
194.1 \\
195.6 \\
197.1\end{array}$ & $\begin{array}{l}9.7 \\
8.7 \\
9.4 \\
9.1 \\
8.1\end{array}$ & $\begin{array}{l}0.2 \\
0.3 \\
0.2 \\
0.2 \\
0.2\end{array}$ & $\begin{array}{l}79 \\
70 \\
76 \\
74 \\
65\end{array}$ \\
\hline $\begin{array}{l}23-1(60.0) \\
23-2(60.0) \\
23-3(60.0) \\
23-4(60.0) \\
23-5(60.0)\end{array}$ & $\begin{array}{l}198.6 \\
200.1 \\
201.6 \\
203.1 \\
204.6\end{array}$ & $\begin{array}{l}8.3 \\
8.8 \\
9.9 \\
6.2 \\
9.0\end{array}$ & $\begin{array}{l}0.4 \\
0.3 \\
0.2 \\
0.2 \\
0.3\end{array}$ & $\begin{array}{l}66 \\
71 \\
81 \\
50 \\
73\end{array}$ \\
\hline $\begin{array}{l}24-2(60.0) \\
24-3(60.0)\end{array}$ & $\begin{array}{l}209.1 \\
210.6\end{array}$ & $\begin{array}{l}6.3 \\
3.9\end{array}$ & $\begin{array}{l}1.2 \\
1.0\end{array}$ & $\begin{array}{l}42 \\
24\end{array}$ \\
\hline
\end{tabular}


TABLE 1 - Continued

\begin{tabular}{|c|c|c|c|c|}
\hline $\begin{array}{l}\text { Core, Section } \\
\text { Top of } \\
\text { Interval } \\
\text { (cm) }\end{array}$ & $\begin{array}{l}\text { Depth } \\
\text { in Hole } \\
(\mathrm{m})\end{array}$ & $\begin{array}{c}\text { Carbon } \\
\text { Total } \\
(\%)\end{array}$ & $\begin{array}{c}\text { Organic } \\
\text { Carbon } \\
(\%)\end{array}$ & $\begin{array}{c}\mathrm{CaCO}_{3} \\
(\%)\end{array}$ \\
\hline $\begin{array}{l}25-2(64.0) \\
25-4(60.0) \\
25-5(60.0) \\
25-6(60.0)\end{array}$ & $\begin{array}{l}218.1 \\
221.1 \\
222.6 \\
224.1\end{array}$ & $\begin{array}{l}5.8 \\
5.5 \\
8.1 \\
7.0\end{array}$ & $\begin{array}{l}0.8 \\
0.7 \\
0.5 \\
0.4\end{array}$ & $\begin{array}{l}42 \\
40 \\
64 \\
55\end{array}$ \\
\hline $\begin{array}{l}26-1(59.0) \\
26-2(60.0) \\
26-3(60.0) \\
26-4(60.0) \\
26-5(60.0) \\
26-6(60.0)\end{array}$ & $\begin{array}{l}225.6 \\
227.1 \\
228.6 \\
230.1 \\
231.6 \\
233.1\end{array}$ & $\begin{array}{l}7.3 \\
8.4 \\
8.0 \\
7.9 \\
8.7 \\
7.7\end{array}$ & $\begin{array}{l}0.8 \\
0.4 \\
0.5 \\
0.5 \\
0.4 \\
0.5\end{array}$ & $\begin{array}{l}54 \\
66 \\
62 \\
62 \\
69 \\
60\end{array}$ \\
\hline $\begin{array}{l}27-1(60.0) \\
27-2(60.0) \\
27-3(60.0) \\
27-4(60.0) \\
27-5(60.0) \\
27-6(60.0)\end{array}$ & $\begin{array}{l}234.6 \\
236.1 \\
237.6 \\
239.1 \\
240.6 \\
242.1\end{array}$ & $\begin{array}{l}7.8 \\
6.4 \\
5.9 \\
6.0 \\
5.9 \\
6.2\end{array}$ & $\begin{array}{l}0.4 \\
0.7 \\
0.7 \\
0.8 \\
0.7 \\
0.9\end{array}$ & $\begin{array}{l}61 \\
48 \\
43 \\
44 \\
43 \\
44\end{array}$ \\
\hline $\begin{array}{l}28-1(70.0) \\
28-2(60.0) \\
28-3(60.0) \\
28-4(60.0) \\
28-5(60.0) \\
28-6(60.0)\end{array}$ & $\begin{array}{l}243.7 \\
245.1 \\
246.6 \\
248.1 \\
249.6 \\
251.1\end{array}$ & $\begin{array}{l}8.3 \\
8.3 \\
8.3 \\
8.3 \\
8.0 \\
9.4\end{array}$ & $\begin{array}{l}0.2 \\
0.2 \\
0.2 \\
0.2 \\
0.3 \\
0.3\end{array}$ & $\begin{array}{l}68 \\
67 \\
68 \\
68 \\
64 \\
76\end{array}$ \\
\hline $\begin{array}{l}29-2(60.0) \\
29-3(60.0)\end{array}$ & $\begin{array}{l}254.1 \\
255.6\end{array}$ & $\begin{array}{l}7.3 \\
5.8\end{array}$ & $\begin{array}{l}0.5 \\
1.0\end{array}$ & $\begin{array}{l}57 \\
41\end{array}$ \\
\hline $\begin{array}{l}30-1(60.0) \\
30-2(60.0) \\
30-3(60.0) \\
30-4(60.0) \\
30-5(60.0) \\
30-6(60.0)\end{array}$ & $\begin{array}{l}261.6 \\
263.1 \\
264.6 \\
266.1 \\
267.6 \\
269.1\end{array}$ & $\begin{array}{l}8.3 \\
9.3 \\
9.4 \\
9.7 \\
9.1 \\
9.2\end{array}$ & $\begin{array}{l}0.3 \\
0.2 \\
0.2 \\
0.2 \\
0.2 \\
0.2\end{array}$ & $\begin{array}{l}67 \\
76 \\
76 \\
79 \\
74 \\
75\end{array}$ \\
\hline $31-1(60.0)$ & 270.6 & 9.9 & 0.2 & 81 \\
\hline $\begin{array}{l}32-1(99.0) \\
32-2(60.0) \\
32-3(60.0) \\
32-4(60.0)\end{array}$ & $\begin{array}{l}280.0 \\
281.1 \\
282.6 \\
284.1\end{array}$ & $\begin{array}{l}8.2 \\
8.9 \\
7.3 \\
9.3\end{array}$ & $\begin{array}{l}0.3 \\
0.3 \\
0.3 \\
0.2\end{array}$ & $\begin{array}{l}65 \\
71 \\
58 \\
76\end{array}$ \\
\hline $\begin{array}{l}33-1(88.0) \\
33-2(60.0)\end{array}$ & $\begin{array}{l}287.9 \\
289.1\end{array}$ & $\begin{array}{l}9.5 \\
9.0\end{array}$ & $\begin{array}{l}0.4 \\
0.3\end{array}$ & $\begin{array}{l}76 \\
73\end{array}$ \\
\hline \multicolumn{5}{|l|}{ DSDP 159} \\
\hline $\begin{array}{l}3-1(5.0) \\
3-3(17.0) \\
3-3(17.0)^{\mathrm{a}} \\
3-3(77.0) \\
3-3(77.0)^{\mathrm{a}}\end{array}$ & $\begin{array}{l}18.0 \\
22.5 \\
22.5 \\
22.0 \\
23.0\end{array}$ & $\begin{array}{l}1.7 \\
0.2 \\
0.1 \\
0.7 \\
0.6\end{array}$ & $\begin{array}{l}0.1 \\
0.1 \\
0.1 \\
0.1 \\
0.1\end{array}$ & $\begin{array}{r}14 \\
1 \\
1 \\
5 \\
4\end{array}$ \\
\hline $4-6(117.0)$ & 35.0 & 1.2 & 0.0 & 10 \\
\hline $5-2(117.0)$ & 44.5 & 3.2 & 0.0 & 27 \\
\hline $6-1(100.0)$ & 73.0 & 4.4 & 0.0 & 36 \\
\hline $9-1(60.0)$ & 72.5 & 8.6 & 0.0 & 72 \\
\hline $13-6(87.0)$ & 108.0 & 4.0 & 0.0 & 33 \\
\hline \multicolumn{5}{|l|}{ DSDP 160} \\
\hline $\begin{array}{l}1-1(29.0) \\
1-2(29.0) \\
1-3(120.0)\end{array}$ & $\begin{array}{l}0.3 \\
1.8 \\
4.2\end{array}$ & $\begin{array}{l}0.4 \\
0.4 \\
0.2\end{array}$ & $\begin{array}{l}0.1 \\
0.1 \\
0.1\end{array}$ & $\begin{array}{l}2 \\
2 \\
1\end{array}$ \\
\hline $\begin{array}{l}2-1(29.0) \\
2-2(29.0) \\
2-3(29.0)\end{array}$ & $\begin{array}{r}9.3 \\
10.8 \\
12.3\end{array}$ & $\begin{array}{l}0.1 \\
0.1 \\
0.2\end{array}$ & $\begin{array}{l}0.1 \\
0.1 \\
0.1\end{array}$ & $\begin{array}{l}0 \\
0 \\
1\end{array}$ \\
\hline
\end{tabular}

${ }^{\mathrm{a}}$ Based on $0.5 \mathrm{~g}$ total carbon sample.
TABLE 1 - Continued

\begin{tabular}{|c|c|c|c|c|}
\hline $\begin{array}{l}\text { Core, Section } \\
\text { Top of } \\
\text { Interval } \\
\text { (cm) }\end{array}$ & $\begin{array}{l}\text { Depth } \\
\text { in Hole } \\
(\mathrm{m})\end{array}$ & $\begin{array}{c}\text { Carbon } \\
\text { Total } \\
(\%)\end{array}$ & $\begin{array}{c}\text { Organic } \\
\text { Carbon } \\
(\%)\end{array}$ & $\begin{array}{c}\mathrm{CaCO}_{3} \\
(\%)\end{array}$ \\
\hline $\begin{array}{l}2-4(29.0) \\
2-5(29.0)\end{array}$ & $\begin{array}{l}13.8 \\
15.3\end{array}$ & $\begin{array}{l}0.1 \\
0.3\end{array}$ & $\begin{array}{l}0.1 \\
0.1\end{array}$ & $\begin{array}{l}0 \\
1\end{array}$ \\
\hline $\begin{array}{l}3-1(120.0) \\
3-2(29.0) \\
3-3(29.0) \\
3-5(29.0) \\
3-6(29.0)\end{array}$ & $\begin{array}{l}19.2 \\
19.8 \\
21.3 \\
24.3 \\
25.8\end{array}$ & $\begin{array}{l}0.2 \\
0.1 \\
0.4 \\
0.1 \\
0.2\end{array}$ & $\begin{array}{l}0.1 \\
0.1 \\
0.1 \\
0.1 \\
0.1\end{array}$ & $\begin{array}{l}1 \\
0 \\
2 \\
0 \\
1\end{array}$ \\
\hline $\begin{array}{l}4-1(80.0) \\
4-2(29.0) \\
4-3(29.0) \\
4-4(29.0) \\
4-5(29.0)\end{array}$ & $\begin{array}{l}27.8 \\
28.8 \\
30.3 \\
31.8 \\
33.3\end{array}$ & $\begin{array}{l}6.7 \\
6.6 \\
8.9 \\
8.4 \\
8.8\end{array}$ & $\begin{array}{l}0.1 \\
0.1 \\
0.1 \\
0.0 \\
0.0\end{array}$ & $\begin{array}{l}55 \\
55 \\
74 \\
70 \\
73\end{array}$ \\
\hline $\begin{array}{l}5-1(70.0) \\
5-2(70.0) \\
5-3(29.0) \\
5-4(29.0) \\
5-5(29.0) \\
5-6(29.0)\end{array}$ & $\begin{array}{l}36.7 \\
38.2 \\
39.3 \\
40.8 \\
42.3 \\
43.8\end{array}$ & $\begin{array}{l}7.2 \\
6.8 \\
8.5 \\
8.4 \\
8.8 \\
8.6\end{array}$ & $\begin{array}{l}0.1 \\
0.0 \\
0.0 \\
0.0 \\
0.0 \\
0.0\end{array}$ & $\begin{array}{l}59 \\
57 \\
71 \\
70 \\
73 \\
72\end{array}$ \\
\hline $\begin{array}{l}6-1(29.0) \\
6-2(29.0) \\
6-3(29.0) \\
6-4(29.0) \\
6-5(29.0) \\
6-6(29.0)\end{array}$ & $\begin{array}{l}45.3 \\
46.8 \\
48.3 \\
49.8 \\
51.3 \\
52.8\end{array}$ & $\begin{array}{r}10.0 \\
8.8 \\
9.5 \\
8.7 \\
9.0 \\
10.7\end{array}$ & $\begin{array}{l}0.0 \\
0.1 \\
0.0 \\
0.0 \\
0.0 \\
0.0\end{array}$ & $\begin{array}{l}83 \\
73 \\
78 \\
72 \\
75 \\
88\end{array}$ \\
\hline $\begin{array}{l}7-1(129.0) \\
7-2(19.0) \\
7-3(29.0) \\
7-4(29.0) \\
7-5(29.0) \\
7-6(29.0)\end{array}$ & $\begin{array}{l}55.3 \\
55.7 \\
57.3 \\
58.8 \\
60.3 \\
61.8\end{array}$ & $\begin{array}{l}10.4 \\
10.2 \\
10.7 \\
10.8 \\
10.5 \\
10.7\end{array}$ & $\begin{array}{l}0.0 \\
0.0 \\
0.0 \\
0.0 \\
0.0 \\
0.0\end{array}$ & $\begin{array}{l}86 \\
85 \\
89 \\
90 \\
87 \\
89\end{array}$ \\
\hline $\begin{array}{l}8-1(29.0) \\
8-2(135.0) \\
8-3(29.0) \\
8-4(29.0) \\
8-5(121.0)\end{array}$ & $\begin{array}{l}63.3 \\
65.8 \\
66.3 \\
67.8 \\
70.2\end{array}$ & $\begin{array}{l}10.9 \\
10.9 \\
11.1 \\
11.0 \\
10.9\end{array}$ & $\begin{array}{l}0.0 \\
0.1 \\
0.0 \\
0.0 \\
0.0\end{array}$ & $\begin{array}{l}91 \\
90 \\
92 \\
91 \\
91\end{array}$ \\
\hline $\begin{array}{l}9-1(29.0) \\
9-2(29.0) \\
9-3(29.0) \\
9-5(29.0) \\
9-6(29.0)\end{array}$ & $\begin{array}{l}72.3 \\
73.8 \\
75.3 \\
78.3 \\
79.8\end{array}$ & $\begin{array}{l}10.8 \\
10.8 \\
10.9 \\
10.6 \\
10.8\end{array}$ & $\begin{array}{l}0.0 \\
0.0 \\
0.0 \\
0.1 \\
0.0\end{array}$ & $\begin{array}{l}90 \\
90 \\
90 \\
87 \\
89\end{array}$ \\
\hline $\begin{array}{l}10-1(40.0) \\
10-2(29.0) \\
10-3(29.0) \\
10-4(29.0) \\
10-5(29.0) \\
10-6(29.0)\end{array}$ & $\begin{array}{l}81.4 \\
82.8 \\
84.3 \\
85.8 \\
87.3 \\
88.8\end{array}$ & $\begin{array}{l}10.4 \\
10.7 \\
10.6 \\
10.4 \\
10.9 \\
11.1\end{array}$ & $\begin{array}{l}0.0 \\
0.1 \\
0.0 \\
0.0 \\
0.0 \\
0.0\end{array}$ & $\begin{array}{l}86 \\
89 \\
88 \\
86 \\
90 \\
92\end{array}$ \\
\hline $\begin{array}{l}11-1(29.0) \\
11-2(29.0) \\
11-3(29.0) \\
11-4(29.0) \\
11-5(20.0) \\
11-6(29.0)\end{array}$ & $\begin{array}{l}90.3 \\
91.8 \\
93.3 \\
94.8 \\
96.2 \\
97.8\end{array}$ & $\begin{array}{l}10.9 \\
10.9 \\
10.8 \\
11.1 \\
10.7 \\
10.9\end{array}$ & $\begin{array}{l}0.0 \\
0.0 \\
0.0 \\
0.0 \\
0.0 \\
0.0\end{array}$ & $\begin{array}{l}91 \\
90 \\
89 \\
92 \\
89 \\
91\end{array}$ \\
\hline $\begin{array}{l}12-1(29.0) \\
12-2(29.0) \\
12-3(29.0) \\
12-4(29.0) \\
12-5(29.0) \\
12-6(29.0)\end{array}$ & $\begin{array}{r}99.3 \\
100.8 \\
102.3 \\
103.8 \\
105.3 \\
106.8\end{array}$ & $\begin{array}{l}10.6 \\
10.5 \\
10.6 \\
10.1 \\
10.5 \\
10.4\end{array}$ & $\begin{array}{l}0.1 \\
0.0 \\
0.0 \\
0.0 \\
0.0 \\
0.0\end{array}$ & $\begin{array}{l}88 \\
87 \\
88 \\
84 \\
87 \\
86\end{array}$ \\
\hline \multicolumn{5}{|l|}{ DSDP 161} \\
\hline $\begin{array}{l}1-1(50.0) \\
1-1(85.0)\end{array}$ & $\begin{array}{l}0.5 \\
0.9\end{array}$ & $\begin{array}{l}0.5 \\
0.2\end{array}$ & $\begin{array}{l}0.1 \\
0.1\end{array}$ & $\begin{array}{l}3 \\
1\end{array}$ \\
\hline
\end{tabular}


TABLE 1 - Continued

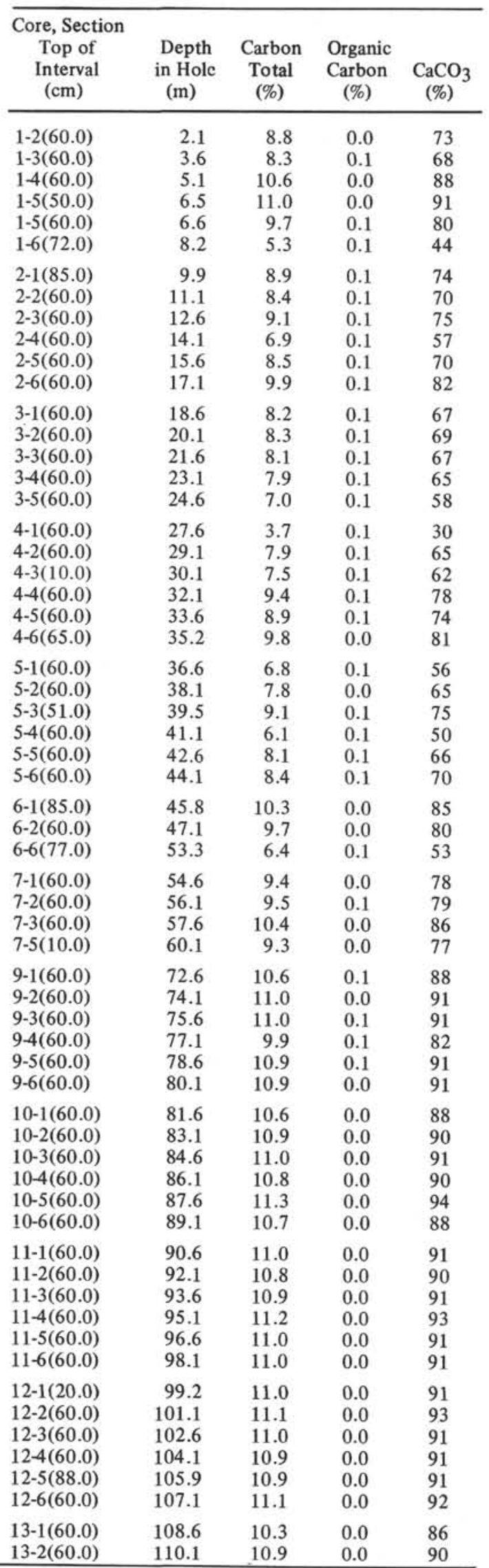

TABLE 1 - Continued

\begin{tabular}{|c|c|c|c|c|}
\hline $\begin{array}{l}\text { Core, Section } \\
\text { Top of } \\
\text { Interval } \\
\text { (cm) }\end{array}$ & $\begin{array}{c}\text { Depth } \\
\text { in Hole } \\
(\mathrm{m})\end{array}$ & $\begin{array}{c}\text { Carbon } \\
\text { Total } \\
(\%)\end{array}$ & $\begin{array}{l}\text { Organic } \\
\text { Carbon } \\
(\%)\end{array}$ & $\begin{array}{c}\mathrm{CaCO}_{3} \\
(\%)\end{array}$ \\
\hline $13-3(60.0)$ & 111.6 & 11.5 & 0.0 & 95 \\
\hline $13-4(60.0)$ & 113.1 & 11.3 & 0.0 & 94 \\
\hline $13-5(60.0)$ & 114.6 & 11.4 & 0.0 & 95 \\
\hline $13-6(60.0)$ & 116.1 & 11.5 & 0.0 & 96 \\
\hline \multicolumn{5}{|l|}{ DSDP 161A } \\
\hline $1-1(50.0)$ & 63.5 & 10.2 & 0.0 & 85 \\
\hline $1-2(50.0)$ & 65.0 & 10.2 & 0.0 & 85 \\
\hline $1-3(60.0)$ & 66.6 & 9.7 & 0.0 & 81 \\
\hline $1-4(60.0)$ & 68.1 & 9.8 & 0.0 & 82 \\
\hline $1-5(70.0)$ & 69.7 & 10.4 & 0.0 & 86 \\
\hline $1-6(50.0)$ & 71.0 & 10.8 & 0.0 & 90 \\
\hline $2-1(60.0)$ & 128.6 & 11.4 & 0.0 & 95 \\
\hline $2-2(60.0)$ & 130.1 & 11.0 & 0.0 & 91 \\
\hline $2-3(60.0)$ & 131.6 & 11.3 & 0.0 & 94 \\
\hline $2-4(60.0)$ & 133.1 & 10.7 & 0.0 & 89 \\
\hline $2-5(60.0)$ & 134.6 & 11.0 & 0.0 & 92 \\
\hline $2-6(50.0)$ & 136.0 & 10.8 & 0.0 & 90 \\
\hline $3-1(60.0)$ & 137.6 & 10.9 & 0.0 & 91 \\
\hline $3-2(50.0)$ & 139.0 & 10.8 & 0.0 & 90 \\
\hline $3-3(55.0)$ & 140.6 & 10.5 & 0.0 & 87 \\
\hline $3-4(60.0)$ & 142.1 & 11.3 & 0.0 & 94 \\
\hline $3-5(60.0)$ & 143.6 & 10.7 & 0.0 & 89 \\
\hline $3-6(60.0)$ & 145.1 & 10.5 & 0.0 & 87 \\
\hline $4-1(59.0)$ & 146.6 & 9.5 & 0.0 & 79 \\
\hline $4-2(60.0)$ & 148.1 & 9.2 & 0.0 & 77 \\
\hline $4-3(60.0)$ & 149.6 & 9.7 & 0.0 & 80 \\
\hline $4-4(42.0)$ & 150.9 & 9.5 & 0.0 & 79 \\
\hline $4-5(60.0)$ & 152.6 & 8.3 & 0.0 & 69 \\
\hline $4-6(65.0)$ & 154.1 & 9.2 & 0.1 & 76 \\
\hline $5-1(60.0)$ & 155.6 & 11.1 & 0.0 & 92 \\
\hline $5-2(60.0)$ & 157.1 & 11.0 & 0.0 & 92 \\
\hline $5-3(60.0)$ & 158.6 & 10.9 & 0.0 & 91 \\
\hline $5-4(60.0)$ & 160.1 & 11.1 & 0.0 & 92 \\
\hline $5-5(60.0)$ & 161.6 & 6.9 & 0.0 & 58 \\
\hline $5-6(60.0)$ & 163.1 & 10.8 & 0.0 & 90 \\
\hline $6-1(60.0)$ & 164.6 & 10.5 & 0.0 & 88 \\
\hline $6-2(60.0)$ & 166.1 & 11.1 & 0.0 & 92 \\
\hline $6-3(60.0)$ & 167.6 & 11.3 & 0.0 & 94 \\
\hline $6-4(60.0)$ & 169.1 & 11.1 & 0.0 & 92 \\
\hline $6-5(60.0)$ & 170.6 & 10.9 & 0.0 & 90 \\
\hline $6-6(60.0)$ & 172.1 & 11.2 & 0.1 & 92 \\
\hline $7-1(60.0)$ & 173.6 & 10.0 & 0.0 & 83 \\
\hline $7-2(60.0)$ & 175.1 & 10.7 & 0.0 & 89 \\
\hline $7-3(25.0)$ & 176.3 & 10.6 & 0.0 & 88 \\
\hline $7-4(50.0)$ & 178.0 & 10.4 & 0.0 & 86 \\
\hline $7-5(60.0)$ & 179.6 & 10.5 & 0.0 & 88 \\
\hline $7-6(60.0)$ & 181.1 & 10.6 & 0.0 & 88 \\
\hline $8-1(60.0)$ & 182.6 & 10.2 & 0.0 & 84 \\
\hline $8-2(60.0)$ & 184.1 & 8.8 & 0.0 & 73 \\
\hline $8-3(60.0)$ & 185.6 & 8.2 & 0.1 & 67 \\
\hline $9-1(107.0)$ & 192.1 & 8.5 & 0.0 & 71 \\
\hline $9-2(60.0)$ & 193.1 & 8.3 & 0.0 & 69 \\
\hline $9-3(60.0)$ & 194.6 & 6.8 & 0.0 & 57 \\
\hline $9-4(60.0)$ & 196.1 & 9.7 & 0.0 & 81 \\
\hline $9-5(60.0)$ & 197.6 & 9.3 & 0.1 & 77 \\
\hline $10-2(53.0)$ & 202.0 & 1.0 & 0.0 & 8 \\
\hline $10-3(41.0)$ & 203.4 & 0.3 & 0.0 & 2 \\
\hline $10-4(59.0)$ & 205.1 & 1.1 & 0.0 & 9 \\
\hline $10-5(60.0)$ & 206.6 & 1.0 & 0.0 & 9 \\
\hline $10-6(60.0)$ & 208.1 & 2.3 & 0.0 & 19 \\
\hline
\end{tabular}


TABLE 1 - Continued

\begin{tabular}{|c|c|c|c|c|}
\hline $\begin{array}{l}\text { Core, Section } \\
\text { Top of } \\
\text { Interval } \\
\text { (cm) }\end{array}$ & $\begin{array}{l}\text { Depth } \\
\text { in Hole } \\
(\mathrm{m})\end{array}$ & $\begin{array}{l}\text { Carbon } \\
\text { Total } \\
(\%)\end{array}$ & $\begin{array}{c}\text { Organic } \\
\text { Carbon } \\
(\%)\end{array}$ & $\underset{(\%)}{\mathrm{CaCO}_{3}}$ \\
\hline $\begin{array}{l}11-1(112.0) \\
11-2(13.0) \\
11-3(60.0) \\
11-4(60.0) \\
11-5(60.0)\end{array}$ & $\begin{array}{l}210.1 \\
210.6 \\
212.6 \\
214.1 \\
215.6\end{array}$ & $\begin{array}{l}2.7 \\
2.4 \\
1.3 \\
2.6 \\
2.2\end{array}$ & $\begin{array}{l}0.0 \\
0.0 \\
0.0 \\
0.0 \\
0.0\end{array}$ & $\begin{array}{l}23 \\
20 \\
11 \\
21 \\
18\end{array}$ \\
\hline $12-1(74.0)$ & 218.7 & 0.8 & 0.0 & 7 \\
\hline $13-1(93.0)$ & 227.9 & 3.9 & 0.0 & 32 \\
\hline $\begin{array}{l}14-1(85.0) \\
14-2(69.0)\end{array}$ & $\begin{array}{l}235.9 \\
237.2\end{array}$ & $\begin{array}{l}6.5 \\
4.2\end{array}$ & $\begin{array}{l}0.1 \\
0.0\end{array}$ & $\begin{array}{l}54 \\
35\end{array}$ \\
\hline \multicolumn{5}{|l|}{ DSDP 162} \\
\hline $\begin{array}{l}1-1(40.0) \\
1-2(40.0) \\
1-3(40.0) \\
1-4(53.0) \\
1-4(60.0) \\
1-5(55.0) \\
1-6(60.0)\end{array}$ & $\begin{array}{l}0.4 \\
1.9 \\
3.4 \\
5.0 \\
5.1 \\
6.6 \\
8.1\end{array}$ & $\begin{array}{r}10.2 \\
7.5 \\
8.9 \\
8.9 \\
10.5 \\
10.0 \\
5.4\end{array}$ & $\begin{array}{l}0.0 \\
0.0 \\
0.0 \\
0.0 \\
0.0 \\
0.0 \\
0.0\end{array}$ & $\begin{array}{l}85 \\
62 \\
74 \\
74 \\
87 \\
83 \\
45\end{array}$ \\
\hline $\begin{array}{l}2-1(60.0) \\
2-2(60.0) \\
2-3(60.0) \\
2-3(60.0) \\
2-5(60.0) \\
2-6(60.0)\end{array}$ & $\begin{array}{r}9.6 \\
11.1 \\
12.6 \\
12.6 \\
15.6 \\
17.1\end{array}$ & $\begin{array}{r}9.1 \\
9.5 \\
10.4 \\
9.4 \\
9.2 \\
9.5\end{array}$ & $\begin{array}{l}0.0 \\
0.0 \\
0.0 \\
0.0 \\
0.3 \\
0.0\end{array}$ & $\begin{array}{l}76 \\
79 \\
87 \\
78 \\
74 \\
79\end{array}$ \\
\hline $\begin{array}{l}3-1(60.0) \\
3-2(60.0) \\
3-3(50.0) \\
3-4(60.0) \\
3-5(60.0) \\
3-6(60.0)\end{array}$ & $\begin{array}{l}18.6 \\
20.1 \\
21.5 \\
23.1 \\
24.6 \\
26.1\end{array}$ & $\begin{array}{l}8.5 \\
6.7 \\
6.9 \\
7.5 \\
8.2 \\
5.0\end{array}$ & $\begin{array}{l}0.2 \\
0.2 \\
0.2 \\
0.9 \\
0.0 \\
0.0\end{array}$ & $\begin{array}{l}69 \\
54 \\
56 \\
55 \\
68 \\
42\end{array}$ \\
\hline $\begin{array}{l}4-1(60.0) \\
4-2(60.0) \\
4-3(60.0) \\
4-4(60.0) \\
4-5(30.0) \\
4-6(7.0)\end{array}$ & $\begin{array}{l}27.6 \\
29.1 \\
30.6 \\
32.1 \\
33.3 \\
34.6\end{array}$ & $\begin{array}{l}9.1 \\
0.4 \\
1.6 \\
0.2 \\
1.2 \\
0.1\end{array}$ & $\begin{array}{l}0.0 \\
0.0 \\
0.0 \\
0.0 \\
0.0 \\
0.0\end{array}$ & $\begin{array}{r}75 \\
3 \\
13 \\
2 \\
10 \\
1\end{array}$ \\
\hline $\begin{array}{l}5-1(90.0) \\
5-2(60.0) \\
5-3(60.0) \\
5-4(60.0) \\
5-5(60.0) \\
5-6(60.0)\end{array}$ & $\begin{array}{l}36.9 \\
38.1 \\
39.6 \\
41.1 \\
42.6 \\
44.1\end{array}$ & $\begin{array}{l}0.2 \\
1.5 \\
1.3 \\
0.0 \\
0.5 \\
0.7\end{array}$ & $\begin{array}{l}0.0 \\
0.0 \\
0.0 \\
0.0 \\
0.0 \\
0.0\end{array}$ & $\begin{array}{r}2 \\
13 \\
11 \\
0 \\
4 \\
5\end{array}$ \\
\hline $\begin{array}{l}6-1(60.0) \\
6-2(6.0) \\
6-3(60.0) \\
6-4(50.0)\end{array}$ & $\begin{array}{l}45.6 \\
46.6 \\
48.6 \\
50.0\end{array}$ & $\begin{array}{l}0.0 \\
0.1 \\
0.2 \\
0.0\end{array}$ & $\begin{array}{l}0.0 \\
0.0 \\
0.0 \\
0.0\end{array}$ & $\begin{array}{l}0 \\
1 \\
1 \\
0\end{array}$ \\
\hline $\begin{array}{l}7-1(30.0) \\
7-2(60.0) \\
7-3(60.0) \\
7-4(60.0) \\
7-5(60.0) \\
7-6(30.0)\end{array}$ & $\begin{array}{l}54.3 \\
56.1 \\
57.6 \\
59.1 \\
60.6 \\
61.8\end{array}$ & $\begin{array}{l}0.1 \\
0.1 \\
0.1 \\
0.0 \\
0.0 \\
0.0\end{array}$ & $\begin{array}{l}0.0 \\
0.0 \\
0.0 \\
0.0 \\
0.0 \\
0.0\end{array}$ & $\begin{array}{l}1 \\
1 \\
1 \\
0 \\
0 \\
0\end{array}$ \\
\hline $\begin{array}{l}8-1(60.0) \\
8-2(60.0) \\
8-3(60.0) \\
8-4(60.0) \\
8-5(60.0) \\
8-6(60.0)\end{array}$ & $\begin{array}{l}63.6 \\
65.1 \\
66.6 \\
68.1 \\
69.6 \\
71.1\end{array}$ & $\begin{array}{l}5.9 \\
6.6 \\
6.0 \\
7.3 \\
6.6 \\
5.3\end{array}$ & $\begin{array}{l}0.0 \\
0.1 \\
0.0 \\
0.0 \\
0.0 \\
0.0\end{array}$ & $\begin{array}{l}49 \\
54 \\
49 \\
61 \\
55 \\
43\end{array}$ \\
\hline $\begin{array}{l}9-1(60.0) \\
9-2(60.0) \\
9-3(60.0)\end{array}$ & $\begin{array}{l}72.6 \\
74.1 \\
75.6\end{array}$ & $\begin{array}{l}6.5 \\
6.8 \\
4.8\end{array}$ & $\begin{array}{l}0.0 \\
0.0 \\
0.1\end{array}$ & $\begin{array}{l}54 \\
57 \\
39 \\
\end{array}$ \\
\hline
\end{tabular}

TABLE 1 - Continued

\begin{tabular}{|c|c|c|c|c|}
\hline $\begin{array}{l}\text { Core, Section } \\
\text { Top of } \\
\text { Interval } \\
\text { (cm) }\end{array}$ & $\begin{array}{l}\text { Depth } \\
\text { in Hole } \\
(\mathrm{m})\end{array}$ & $\begin{array}{c}\text { Carbon } \\
\text { Total } \\
(\%)\end{array}$ & $\begin{array}{c}\text { Organic } \\
\text { Carbon } \\
(\%)\end{array}$ & $\begin{array}{c}\mathrm{CaCO}_{3} \\
(\%)\end{array}$ \\
\hline $\begin{array}{l}9-4(60.0) \\
9-5(60.0) \\
9-6(60.0)\end{array}$ & $\begin{array}{l}77.1 \\
78.6 \\
80.1\end{array}$ & $\begin{array}{l}6.4 \\
1.0 \\
2.0\end{array}$ & $\begin{array}{l}0.0 \\
0.0 \\
0.1\end{array}$ & $\begin{array}{r}53 \\
8 \\
16\end{array}$ \\
\hline $\begin{array}{l}10-2(60.0) \\
10-3(10.0) \\
10-4(60.0) \\
10-5(20.0) \\
10-6(60.0)\end{array}$ & $\begin{array}{l}83.1 \\
84.1 \\
86.1 \\
87.2 \\
89.1\end{array}$ & $\begin{array}{l}2.1 \\
0.3 \\
2.9 \\
2.3 \\
2.5\end{array}$ & $\begin{array}{l}0.1 \\
0.0 \\
0.0 \\
0.0 \\
0.0\end{array}$ & $\begin{array}{r}17 \\
2 \\
24 \\
19 \\
20\end{array}$ \\
\hline $\begin{array}{l}11-1(126.0) \\
11-2(60.0) \\
11-3(52.0) \\
11-4(60.0) \\
11-5(60.0) \\
11-6(60.0)\end{array}$ & $\begin{array}{l}91.3 \\
92.1 \\
93.5 \\
95.1 \\
96.6 \\
98.1\end{array}$ & $\begin{array}{l}1.1 \\
2.6 \\
2.7 \\
3.1 \\
1.8 \\
3.0\end{array}$ & $\begin{array}{l}0.0 \\
0.1 \\
0.0 \\
0.5 \\
0.1 \\
0.3\end{array}$ & $\begin{array}{r}9 \\
21 \\
22 \\
22 \\
15 \\
22\end{array}$ \\
\hline $\begin{array}{l}12-1(60.0) \\
12-2(60.0) \\
12-4(60.0) \\
12-3(60.0) \\
12-5(60.0) \\
12-6(60.0)\end{array}$ & \begin{tabular}{r|}
99.6 \\
101.1 \\
104.1 \\
102.6 \\
105.6 \\
107.1
\end{tabular} & $\begin{array}{l}0.9 \\
1.0 \\
0.3 \\
1.1 \\
1.3 \\
3.1\end{array}$ & $\begin{array}{l}0.0 \\
0.0 \\
0.0 \\
0.0 \\
0.0 \\
0.0\end{array}$ & $\begin{array}{r}7 \\
8 \\
3 \\
9 \\
11 \\
25\end{array}$ \\
\hline $\begin{array}{l}13-1(60.0) \\
13-2(60.0) \\
13-3(60.0) \\
13-4(60.0) \\
13-5(60.0) \\
13-6(60.0)\end{array}$ & $\begin{array}{l}108.6 \\
110.1 \\
111.6 \\
113.1 \\
114.6 \\
116.1\end{array}$ & $\begin{array}{l}3.0 \\
5.5 \\
4.7 \\
2.8 \\
5.1 \\
0.7\end{array}$ & $\begin{array}{l}0.0 \\
0.0 \\
0.0 \\
0.0 \\
0.0 \\
0.0\end{array}$ & $\begin{array}{r}25 \\
45 \\
39 \\
23 \\
42 \\
6\end{array}$ \\
\hline $\begin{array}{l}14-1(60.0) \\
14-2(60.0) \\
14-3(60.0) \\
14-4(60.0) \\
14-5(60.0) \\
14-6(60.0)\end{array}$ & $\begin{array}{l}117.6 \\
119.1 \\
120.6 \\
122.1 \\
123.6 \\
125.1\end{array}$ & $\begin{array}{l}2.1 \\
1.3 \\
2.4 \\
4.4 \\
1.6 \\
3.5\end{array}$ & $\begin{array}{l}0.1 \\
0.0 \\
0.0 \\
0.1 \\
0.0 \\
0.0\end{array}$ & $\begin{array}{l}17 \\
10 \\
19 \\
36 \\
13 \\
29\end{array}$ \\
\hline $\begin{array}{l}15-1(60.0) \\
15-2(60.0) \\
15-3(10.0) \\
15-4(60.0) \\
15-5(60.0) \\
15-6(60.0)\end{array}$ & $\begin{array}{l}126.6 \\
128.1 \\
129.1 \\
131.1 \\
132.6 \\
134.1\end{array}$ & $\begin{array}{l}3.8 \\
2.3 \\
2.9 \\
4.1 \\
4.2 \\
4.4\end{array}$ & $\begin{array}{l}0.0 \\
0.0 \\
0.0 \\
0.0 \\
0.0 \\
0.0\end{array}$ & $\begin{array}{l}31 \\
19 \\
24 \\
34 \\
34 \\
36\end{array}$ \\
\hline $\begin{array}{l}16-1(70.0) \\
16-2(60.0) \\
16-3(42.0)\end{array}$ & $\begin{array}{l}135.7 \\
137.1 \\
138.4\end{array}$ & $\begin{array}{l}2.2 \\
0.0 \\
0.0\end{array}$ & $\begin{array}{l}0.1 \\
0.0 \\
0.1\end{array}$ & $\begin{array}{r}18 \\
0 \\
0\end{array}$ \\
\hline $\begin{array}{l}17-1(22.0) \\
17-2(60.0) \\
17-3(14.0) \\
17-4(107.0)\end{array}$ & $\begin{array}{l}144.2 \\
146.1 \\
147.1 \\
149.6\end{array}$ & $\begin{array}{l}0.0 \\
8.6 \\
6.2 \\
9.5\end{array}$ & $\begin{array}{l}0.1 \\
0.1 \\
0.1 \\
0.1\end{array}$ & $\begin{array}{r}0 \\
71 \\
51 \\
79\end{array}$ \\
\hline \multicolumn{5}{|l|}{ DSDP 163} \\
\hline $\begin{array}{l}2-1(103.0) \\
2-2(50.0) \\
2-3(50.0) \\
2-4(50.0)\end{array}$ & $\begin{array}{l}2.0 \\
3.0 \\
4.5 \\
6.0\end{array}$ & $\begin{array}{l}0.1 \\
0.1 \\
0.0 \\
0.0\end{array}$ & $\begin{array}{l}0.1 \\
0.1 \\
0.0 \\
0.0\end{array}$ & $\begin{array}{l}0 \\
0 \\
0 \\
0\end{array}$ \\
\hline $\begin{array}{l}3-1(26.0) \\
3-2(50.0) \\
3-3(50.0) \\
3-4(70.0) \\
3-5(50.0) \\
3-6(440.0)\end{array}$ & $\begin{array}{l}10.3 \\
12.0 \\
13.5 \\
15.2 \\
16.5 \\
21.9\end{array}$ & $\begin{array}{l}0.0 \\
0.0 \\
0.0 \\
0.0 \\
0.3 \\
0.1\end{array}$ & $\begin{array}{l}0.0 \\
0.0 \\
0.0 \\
0.0 \\
0.1 \\
0.1\end{array}$ & $\begin{array}{l}0 \\
0 \\
0 \\
0 \\
2 \\
0\end{array}$ \\
\hline $\begin{array}{l}4-1(50.0) \\
4-2(50.0) \\
4-3(50.0) \\
4-4(50.0) \\
4-6(20.0)\end{array}$ & $\begin{array}{l}19.5 \\
21.0 \\
22.5 \\
24.0 \\
26.7\end{array}$ & $\begin{array}{l}0.1 \\
0.1 \\
0.1 \\
0.1 \\
0.3\end{array}$ & $\begin{array}{l}0.0 \\
0.0 \\
0.0 \\
0.0 \\
0.0\end{array}$ & $\begin{array}{l}1 \\
0 \\
0 \\
0 \\
2\end{array}$ \\
\hline
\end{tabular}


TABLE 1 - Continued

\begin{tabular}{|c|c|c|c|c|}
\hline $\begin{array}{l}\text { Core, Section } \\
\text { Top of } \\
\text { Interval } \\
\text { (cm) }\end{array}$ & $\begin{array}{c}\text { Depth } \\
\text { in Hole } \\
\text { (m) }\end{array}$ & $\begin{array}{c}\text { Carbon } \\
\text { Total } \\
(\%)\end{array}$ & $\begin{array}{c}\text { Organic } \\
\text { Carbon } \\
(\%)\end{array}$ & $\begin{array}{c}\mathrm{CaCO}_{3} \\
(\%)\end{array}$ \\
\hline $5-1(50.0)$ & 28.5 & 0.0 & 0.0 & 0 \\
\hline $5-2(50.0)$ & 30.0 & 0.0 & 0.0 & 0 \\
\hline $5-3(50.0)$ & 31.5 & 0.0 & 0.0 & 0 \\
\hline $5-4(50.0)$ & 33.0 & 0.0 & 0.0 & 0 \\
\hline $5-5(50.0)$ & 34.5 & 0.1 & 0.1 & 0 \\
\hline $6-2(50.0)$ & 39.0 & 0.0 & 0.0 & 0 \\
\hline $6-3(50.0)$ & 40.5 & 0.0 & 0.0 & 0 \\
\hline $6-4(50.0)$ & 42.0 & 0.0 & 0.0 & 0 \\
\hline $6-5(50.0)$ & 43.5 & 0.0 & 0.0 & 0 \\
\hline $6-6(50.0)$ & 45.0 & 0.1 & 0.0 & 1 \\
\hline $7-1(50.0)$ & 46.5 & 0.1 & 0.0 & 1 \\
\hline $7-2(50.0)$ & 48.0 & 0.1 & 0.0 & 1 \\
\hline $7-3(50.0)$ & 49.5 & 0.1 & 0.0 & 0 \\
\hline $7-4(50.0)$ & 51.0 & 0.0 & 0.0 & 0 \\
\hline $7-5(50.0)$ & 52.5 & 0.1 & 0.0 & 0 \\
\hline $7-6(50.0)$ & 54.0 & 0.0 & 0.0 & 0 \\
\hline $9-1(26.0)$ & 64.3 & 0.1 & 0.1 & 0 \\
\hline $9-3(50.0)$ & 67.5 & 0.1 & 0.1 & 0 \\
\hline $10-1(64.0)$ & 73.6 & 0.0 & 0.0 & 0 \\
\hline $10-2(40.0)$ & 74.9 & 0.0 & 0.0 & 0 \\
\hline $10-3(50.0)$ & 76.5 & 0.1 & 0.0 & 1 \\
\hline $10-4(50.0)$ & 78.0 & 0.0 & 0.0 & 0 \\
\hline $10-5(50.0)$ & 79.5 & 0.0 & 0.0 & 0 \\
\hline $10-6(50.0)$ & 81.0 & 0.0 & 0.0 & 0 \\
\hline $11-1(50.0)$ & 82.5 & 0.0 & 0.0 & 0 \\
\hline $11-2(50.0)$ & 84.0 & 0.1 & 0.0 & 0 \\
\hline $11-3(50.0)$ & 85.5 & 0.0 & 0.0 & 0 \\
\hline $11-4(50.0)$ & 87.0 & 0.0 & 0.0 & 0 \\
\hline $11-5(50.0)$ & 88.5 & 0.0 & 0.0 & 0 \\
\hline $11-6(50.0)$ & 90.0 & 0.0 & 0.0 & 0 \\
\hline $12-1(50.0)$ & 91.5 & 0.0 & 0.0 & 0 \\
\hline $12-2(50.0)$ & 93.0 & 0.0 & 0.0 & 0 \\
\hline $12-3(50.0)$ & 94.5 & 0.1 & 0.1 & 0 \\
\hline $16-1(50.0)$ & 171.5 & 9.1 & 0.0 & 75 \\
\hline $16-2(50.0)$ & 173.0 & 8.9 & 0.0 & 74 \\
\hline $16-3(50.0)$ & 174.5 & 9.1 & 0.0 & 76 \\
\hline $16-4(50.0)$ & 176.0 & 9.7 & 0.0 & 81 \\
\hline $16-5(50.0)$ & 177.5 & 8.3 & 0.0 & 69 \\
\hline $16-6(50.0)$ & 179.0 & 7.0 & 0.0 & 59 \\
\hline $15-1(22.0)$ & 162.2 & 2.7 & 0.0 & 23 \\
\hline $15-6(106.0)$ & 170.6 & 7.9 & 0.0 & 66 \\
\hline $17-1(50.0)$ & 180.5 & 7.5 & 0.0 & 63 \\
\hline $17-2(50.0)$ & 182.0 & 8.6 & 0.0 & 72 \\
\hline $17-3(50.0)$ & 183.5 & 5.4 & 0.0 & 45 \\
\hline $17-4(50.0)$ & 185.0 & 8.3 & 0.0 & 69 \\
\hline $17-5(50.0)$ & 186.5 & 8.7 & 0.0 & 72 \\
\hline $17-6(50.0)$ & 188.0 & 8.3 & 0.0 & 69 \\
\hline $18-1(50.0)$ & 189.5 & 8.8 & 0.0 & 73 \\
\hline $18-2(50.0)$ & 191.0 & 6.3 & 0.0 & 52 \\
\hline $18-3(40.0)$ & 192.4 & 5.5 & 0.0 & 45 \\
\hline $18-4(50.0)$ & 194.0 & 8.1 & 0.0 & 68 \\
\hline $18-6(50.0)$ & 197.0 & 10.6 & 0.0 & 88 \\
\hline $19-1(50.0)$ & 198.5 & 9.6 & 0.0 & 80 \\
\hline $19-2(115.0)$ & 200.6 & 5.8 & 0.0 & 49 \\
\hline $19-3(50.0)$ & 201.5 & 7.5 & 0.0 & 62 \\
\hline $19-4(50.0)$ & 203.0 & 10.2 & 0.0 & 85 \\
\hline $19-5(50.0)$ & 204.5 & 10.2 & 0.0 & 85 \\
\hline $19-6(50.0)$ & 206.0 & 10.1 & 0.1 & 84 \\
\hline $20-1(50.0)$ & 207.5 & 8.6 & 0.0 & 72 \\
\hline $20-2(50.0)$ & 209.0 & 9.7 & 0.0 & 81 \\
\hline $20-3(50.0)$ & 210.5 & 10.0 & 0.0 & 83 \\
\hline
\end{tabular}

TABLE 1 - Continued

\begin{tabular}{|c|c|c|c|c|}
\hline $\begin{array}{l}\text { Core, Section } \\
\text { Top of } \\
\text { Interval } \\
\text { (cm) }\end{array}$ & $\begin{array}{c}\text { Depth } \\
\text { in Hole } \\
\text { (m) }\end{array}$ & $\begin{array}{c}\text { Carbon } \\
\text { Total } \\
(\%)\end{array}$ & $\begin{array}{c}\text { Organic } \\
\text { Carbon } \\
(\%)\end{array}$ & $\begin{array}{c}\mathrm{CaCO}_{3} \\
(\%)\end{array}$ \\
\hline $\begin{array}{l}20-4(50.0) \\
20-5(50.0) \\
20-6(50.0)\end{array}$ & $\begin{array}{l}212.0 \\
213.5 \\
215.0\end{array}$ & $\begin{array}{r}9.5 \\
9.2 \\
10.6\end{array}$ & $\begin{array}{l}0.0 \\
0.0 \\
0.0\end{array}$ & $\begin{array}{l}79 \\
77 \\
88\end{array}$ \\
\hline $\begin{array}{l}21-2(50.0) \\
21-3(50.0) \\
21-4(50.0) \\
21-5(50.0) \\
21-6(50.0)\end{array}$ & $\begin{array}{l}218.0 \\
219.5 \\
221.0 \\
222.5 \\
224.0\end{array}$ & $\begin{array}{l}11.4 \\
10.7 \\
10.6 \\
10.9 \\
10.5\end{array}$ & $\begin{array}{l}0.0 \\
0.0 \\
0.0 \\
0.0 \\
0.0\end{array}$ & $\begin{array}{l}95 \\
89 \\
88 \\
90 \\
87\end{array}$ \\
\hline $\begin{array}{l}22-1(97.0) \\
22-2(50.0) \\
22-3(50.0) \\
22-4(50.0) \\
22-5(50.0) \\
22-6(10.0)\end{array}$ & $\begin{array}{l}226.0 \\
227.0 \\
228.5 \\
230.0 \\
231.5 \\
232.6\end{array}$ & $\begin{array}{r}11.1 \\
11.0 \\
9.5 \\
9.9 \\
10.6 \\
9.0\end{array}$ & $\begin{array}{l}0.0 \\
0.0 \\
0.0 \\
0.0 \\
0.0 \\
0.0\end{array}$ & $\begin{array}{l}92 \\
91 \\
79 \\
83 \\
88 \\
75\end{array}$ \\
\hline $\begin{array}{l}23-1(50.0) \\
23-2(50.0) \\
23-3(50.0) \\
23-4(50.0) \\
23-5(50.0) \\
23-6(50.0)\end{array}$ & $\begin{array}{l}234.5 \\
236.0 \\
237.5 \\
239.0 \\
240.5 \\
242.0\end{array}$ & $\begin{array}{l}11.1 \\
10.6 \\
10.9 \\
10.6 \\
10.7 \\
10.1\end{array}$ & $\begin{array}{l}0.0 \\
0.0 \\
0.0 \\
0.0 \\
0.0 \\
0.0\end{array}$ & $\begin{array}{l}92 \\
88 \\
91 \\
88 \\
89 \\
84\end{array}$ \\
\hline $24-1(91.0)$ & 243.9 & 10.6 & 0.0 & 88 \\
\hline $25-1(77.0)$ & 252.8 & 10.1 & 0.0 & 84 \\
\hline $\begin{array}{l}26-1(111.0) \\
26-2(50.0)\end{array}$ & $\begin{array}{l}262.1 \\
263.0\end{array}$ & $\begin{array}{r}9.5 \\
10.4\end{array}$ & $\begin{array}{l}0.0 \\
0.0\end{array}$ & $\begin{array}{l}78 \\
86\end{array}$ \\
\hline $27-1(50.0)$ & 270.5 & 9.7 & 0.0 & 80 \\
\hline \multicolumn{5}{|l|}{ DSDP 163A } \\
\hline $\begin{array}{l}1-2(78.0) \\
1-3(50.0) \\
1-4(50.0)\end{array}$ & $\begin{array}{l}142.3 \\
143.5 \\
145.0\end{array}$ & $\begin{array}{l}0.1 \\
0.0 \\
0.1\end{array}$ & $\begin{array}{l}0.0 \\
0.0 \\
0.0\end{array}$ & $\begin{array}{l}0 \\
0 \\
0\end{array}$ \\
\hline
\end{tabular}

TABLE 2

Gravimetric Determinations

of Calcium Carbonate in

Leg 16 Samples

\begin{tabular}{|c|c|c|c|}
\hline $\begin{array}{l}\text { Core, Section } \\
\text { Interval }(\mathrm{cm})\end{array}$ & $\begin{array}{c}\mathrm{CaCO}_{3} \\
(\%)\end{array}$ & $\begin{array}{l}\text { Core, Section } \\
\text { Interval }(\mathrm{cm})\end{array}$ & $\begin{array}{c}\mathrm{CaCO}_{3} \\
(\%)\end{array}$ \\
\hline DSDP 159 & & DSDP 161A & \\
\hline $2-1(38-43)$ & 2.4 & $10-2(83-86)$ & 13.8 \\
\hline $3-4(78-83)$ & 1.6 & $11-4(97-102)$ & 24.5 \\
\hline $6-3(91-96)$ & 45.6 & $12-1(59-64)$ & 7.5 \\
\hline $9-4(58-63)$ & 66.5 & $12-1(139-144)$ & 12.3 \\
\hline $10-2(5-10)$ & 87.9 & $13-1(136-141)$ & 3.7 \\
\hline $10-6(90-95)$ & 61.8 & $14-1(148-150)$ & 55.9 \\
\hline $12-1(25-30)$ & 67.3 & $14-2(149-151)$ & 13.2 \\
\hline $12-2(15-20)$ & 69.9 & & \\
\hline $12-6(103-108)$ & 73.3 & DSDP 162 & \\
\hline $13-2(95-100)$ & 62.5 & $12-2(104-109)$ & 20.7 \\
\hline $13-4(133-137)$ & 62.5 & $14-2(47-52)$ & 10.9 \\
\hline & & $16-1(108-113)$ & 4.5 \\
\hline DSDP 160 & & $16-3(76-81)$ & 2.7 \\
\hline $13-1(99-103)$ & 29.7 & $17-1(5-10)$ & 5.7 \\
\hline $3-4(78-83)$ & 6.9 & $17-1(62-67)$ & 8.9 \\
\hline $3-5(100-105)$ & 2.0 & $17-4(101-106)$ & 73.8 \\
\hline $6-5(20-25)$ & 78.7 & & \\
\hline $10-6(125-130)$ & 91.8 & DSDP 163 & \\
\hline $11-6(135-140)$ & 87.9 & $2-3(130-135)$ & 8.5 \\
\hline $12-5(135-140)$ & 86.1 & $6-5(115-120)$ & 7.4 \\
\hline
\end{tabular}




\section{DSDP CARBON DETERMINATIONS}

Each sample was ground to a homogeneous fine powder, dried 24 hours at $105-110^{\circ} \mathrm{C}$, divided into two parts, and weighed. The first part was analyzed for its total carbon content, and the second part was acidified with hydrochloric acid and then analyzed for residual carbon which is labeled in the table as "organic" carbon. The carbon contents are reported in per cent by weight. The percentages of calcium carbonate in the sample are calculated as follows: (\% total $\mathrm{C}-\% \mathrm{C}$ after acidification) $\times 8.33=$ per cent calcium carbonate $\left(\mathrm{CaCO}_{3}\right)$. Evidently, the sediments may contain magnesium carbonate, iron carbonate, and perhaps other carbonates as well. In those cases, the percentage carbonate listed refer to more than calcium carbonate alone.

The carbonate determinations were made with a LECO (Laboratory Equipment Corporation) carbon analyzer. A detailed description of the technique can be found in the references given in the introduction. The standard equipment used is the LECO 70 Second Carbon Analyzer; precisions are as follows:

Total Carbon

(1.2 to $12 \%): \pm 0.2 \%$ (absolute variation)

( 0 to $1.2 \%): \pm 0.04 \%$ (absolute variation)

Organic Carbon $\pm 0.04 \%$ (absolute variation)

Calcium Carbonate

(10 to $100 \%): \pm 2.0 \%$ (absolute variation)

(0 to $10 \%$ ): $\pm 0.6 \%$ (absolute variation)

With a single operator and over a short period of time, the standard deviation for calcium carbonate between 10 and 100 per cent is \pm 0.2 per cent. Over a longer period of time and with more than one operator, the standard deviation is about \pm 0.6 per cent. For data between 0 and 10 per cent, the standard deviation is about \pm 0.15 per cent.

Due to mechanical and electronic difficulties, the LECO 70 Second Analyzer was inoperative during part of the analysis program, and a second method, the LECO Acid-Base Semi-Automatic Carbon Determinator, was substituted. The procedure is described in Boyce and Bode (1972). In general, this technique compares well in precision and accuracy with the LECO 70 Second Analyzer, but it is possible that a slight degradation of the data may have resulted in the present case.

A key to the different analysis methods used precedes Table 1.

\section{GRAVIMETRIC ANALYSES}

For a number of samples that were studied in connection with the report on iron-rich basal sediments (Chapter 18 , this volume), analyses of calcium carbonate were made in the laboratory of D.S. Cronan, using a gravimetric technique. Each sample was finely ground and heated to $105^{\circ} \mathrm{C}$ to remove moisture. Approximately $0.25-0.50 \mathrm{gm}$ of material was weighed into a $150 \mathrm{ml}$ Erlenmeyer flask. Carbon dioxide was evolved by treatment with $\mathrm{H}_{3} \mathrm{PO}_{4}$, bubbled through $\mathrm{H}_{2} \mathrm{SO}_{4}$ under right vacuum, and absorbed in Ascarite in two weighed $U$ tubes. The results, calculated as calcium carbonate, are shown in Table 2. This data has not been included in graphic summaries in the site descriptions.

\section{REFERENCES}

Bader, R.G., Gerard, R.D. et al., 1970. Initial Reports of the Deep Sea Drilling Project, Volume IV. Washington (U.S. Government Printing Office). 745.

Boyce, R.E. and Bode, G.W., 1972. Carbon and carbonate analyses, Leg 9, Deep Sea Drilling Project. In Hays et al., 1972. Initial Reports of the Deep Sea Drilling Project, Volume IX. Washington (U.S. Government Printing Office). 797.

\section{KEY TO CARBON-CARBONATE ANALYSIS METHODS}

\section{Organic Carbon Analysis by Acid-Base Method}

$157-22-3(60 \mathrm{~cm})$ through $157-33-2(60 \mathrm{~cm})$.

$157-33-4(60 \mathrm{~cm})$ through $157-42-1(70 \mathrm{~cm})$.

$157-\mathrm{A}-1-2(30 \mathrm{~cm})$ through $157 \mathrm{~A}-3-4(60 \mathrm{~cm})$.

$158-1-2(60 \mathrm{~cm})$ through $158-1-3(60 \mathrm{~cm})$.

$158-6-2(60 \mathrm{~cm})$.

$158-10-5(60 \mathrm{~cm})$.

$158-21-4(60 \mathrm{~cm})$.

$158-24-2(60 \mathrm{~cm})$.

$158-27-5(60 \mathrm{~cm})$.

$160-2-1(29 \mathrm{~cm})$.

$160-2-4(29 \mathrm{~cm})$.

$160-3-5(29 \mathrm{~cm})$.

$160-6-5(29 \mathrm{~cm})$.

$161-1-1(85 \mathrm{~cm})$.

\section{Total Carbon and Organic Carbon By Acid-Base Method}

$157 \mathrm{~A}-3-5(70 \mathrm{~cm})$ through $158-1-1(60 \mathrm{~cm})$. $158-2-1(60 \mathrm{~cm})$ through $158-6-1(60 \mathrm{~cm})$. $158-6-3(60 \mathrm{~cm})$ through $158-10-4(60 \mathrm{~cm})$. $158-10-6(60 \mathrm{~cm})$ through $158-15-5(60 \mathrm{~cm})$. $158-16-1(60 \mathrm{~cm})$ through $158-19-4(60 \mathrm{~cm})$. $158-19-6(60 \mathrm{~cm})$ through $158-21-3(60 \mathrm{~cm})$. $158-21-5(60 \mathrm{~cm})$ through $158-23-5(60 \mathrm{~cm})$. $158-25-2(64 \mathrm{~cm})$ through $158-27-4(60 \mathrm{~cm})$. $158-27-6(60 \mathrm{~cm})$ through $158-9-2(60 \mathrm{~cm})$. $158-30-1(60 \mathrm{~cm})$ through $158-32-2(60 \mathrm{~cm})$. $158-32-4(60 \mathrm{~cm})$ through $160-1-3(120 \mathrm{~cm})$. $160-2-3(29 \mathrm{~cm})$. $160-2-5(29 \mathrm{~cm})$ through $160-3-3(29 \mathrm{~cm})$. $160-4-1(80 \mathrm{~cm})$ through $160-6-4(29 \mathrm{~cm})$. $160-6-6(29 \mathrm{~cm})$ through $161-1-1(50 \mathrm{~cm})$. $161-1-2(60 \mathrm{~cm})$ through $161-12-3(60 \mathrm{~cm})$.

\section{Total Carbon By Acid-Base Method}

\author{
$158-15-6(60 \mathrm{~cm})$. \\ $158-19-5(100 \mathrm{~cm})$. \\ $158-29-3(60 \mathrm{~cm})$. \\ $158-32-3(60 \mathrm{~cm})$. \\ $161-12-4(60 \mathrm{~cm})$ through $161 \mathrm{~A}-1-4(60 \mathrm{~cm})$. \\ $161 \mathrm{~A}-1-6(50 \mathrm{~cm})$ through $161 \mathrm{~A}-7-5(60 \mathrm{~cm})$.
}

